\title{
Comparison of Single and Repeated Dosing of Anti-Inflammatory Human Umbilical Cord Mesenchymal Stromal Cells in a Mouse Model of Polymicrobial Sepsis
}

\author{
Barbara Fazekas $^{1} \cdot$ Senthilkumar Alagesan ${ }^{2} \cdot$ Luke Watson $^{2} \cdot$ Olivia Ng $^{1,2}$. Callum M. Conroy ${ }^{1,2} \cdot$ Cristina Català $^{3}$. \\ Maria Velascode Andres ${ }^{3} \cdot$ Neema Negi $^{1} \cdot$ Jared Q. Gerlach ${ }^{4} \cdot$ Sean O. Hynes ${ }^{5,6}$ • Francisco Lozano ${ }^{3,7,8}$. \\ Stephen J. Elliman ${ }^{2} \cdot$ Matthew D. Griffin ${ }^{1,9,10}$
}

Accepted: 22 December 2021 / Published online: 10 January 2022

(c) The Author(s) 2022

Summary Mesenchymal stromal cells (MSCs) ameliorate pre-clinical sepsis and sepsis-associated acute kidney injury (SAAKI) but clinical trials of single-dose MSCs have not indicated robust efficacy. This study investigated immunomodulatory effects of a novel MSC product (CD362-selected human umbilical cord-derived MSCs [hUC-MSCs]) in mouse endotoxemia and polymicrobial sepsis models. Initially, mice received intra-peritoneal (i.p.) lipopolysaccharide (LPS) followed by single i.p. doses of hUC-MSCs or vehicle. Next, mice underwent cecal ligation and puncture (CLP) followed by intravenous (i.v.) doses of hUC-MSCs at $4 \mathrm{~h}$ or 4 and $28 \mathrm{~h}$. Analyses included serum/plasma assays of biochemical indices, inflammatory mediators and the AKI biomarker NGAL; multi-color flow cytometry of peritoneal macrophages (LPS) and intra-renal immune cell subpopulations (CLP) and histology/immunohistochemistry of kidney (CLP). At 72 h post-LPS injections, hUC-MSCs reduced serum inflammatory mediators and peritoneal macrophage M1/M2 ratio. Repeated, but not single, hUC-MSC doses administered at $48 \mathrm{~h}$ post-CLP resulted in lower serum concentrations of inflammatory mediators, lower plasma NGAL and reversal of sepsis-associated depletion of intra-renal T cell and myeloid cell subpopulations. Hierarchical clustering analysis of all 48-h serum/plasma analytes demonstrated partial co-clustering of repeated-dose hUC-MSC CLP animals with a Sham group but did not reveal a distinct signature of response to therapy. It was concluded that repeated doses of CD362-selected hUC-MSCs are required to modulate systemic and local immune/inflammatory events in polymicrobial sepsis and SA-AKI. Inter-individual variability and lack of effect of single dose MSC administration in the CLP model are consistent with observations to date from early-phase clinical trials.

Keywords Acute kidney injury $\cdot$ Mesenchymal stromal cell $\cdot$ Regenerative medicine $\cdot$ Sepsis $\cdot$ Inflammation $\cdot$ Cell therapy

Barbara Fazekas and Senthilkumar Alagesan contributed equally to this work.

Matthew D. Griffin

matthew.griffin@nuigalway.ie

1 Regenerative Medicine Institute at CÚRAM Centre for Research in Medical Devices, School of Medicine, National University of Ireland Galway, Galway, Ireland

2 Orbsen Therapeutics Ltd., Galway, Ireland

3 Institut d'Investigacions Biomèdiques August Pi i Sunyer, Barcelona, Spain

4 Glycoscience Group, National Centre for Biomedical Engineering Science, National University of Ireland Galway, Galway, Ireland

5 Discipline of Pathology, School of Medicine, National University of Ireland Galway, Galway, Ireland
6 Department of Histopathology, Galway University Hospitals, Galway, Ireland

7 Servei d'Immunologia, Hospital Clínic de Barcelona, Barcelona, Spain

8 Department de Biomedicina, Universitat de Barcelona, Barcelona, Spain

9 Department of Nephrology, Saolta University Health Care Group, Galway University Hospitals, Galway, Ireland

10 National University of Ireland Galway, REMEDI, Biomedical Sciences, Corrib Village, Dangan, Galway H91 TK33, Ireland 


\section{Abbreviations}

SA-AKI

hUC-MSC

LPS

CLP

Intravenous

Intraperitoneal

NGAL

Mesenchymal stromal cells

Sepsis-associated acute kidney injury

Human umbilical cord-derived MSC

Lipopolysaccharide

Cecal ligation and puncture

i.v.

i.p.

neutrophil gelatinase-associated lipocalin

\section{Introduction}

Sepsis is characterised by life-threatening organ dysfunction due to a dysregulated host response to infection caused by bacterial, fungal, viral, and parasitic pathogens [1]. Each year, sepsis affects nearly 50 million people worldwide, frequently leading to severe systemic consequences and organ injuries, including acute kidney injury (AKI), resulting in approximately 11 million deaths [2,3]. Mesenchymal stromal cells (MSC) are multipotent cells with extensive immune-modulatory properties that can be isolated from various tissues [4]. It is now well recognised that MSC offer a potential disease-modulating therapy for sepsis and sepsis-associated AKI (SA-AKI) [5, 6]. Several recent studies using mouse and rat cecal ligation and puncture (CLP), lipopolysaccharide (LPS) or fecal peritonitis models have shown that systemic administration of MSC has the potential to reduce inflammation, counteract bacterial infection and improve the repair of injured tissue in sepsis [7-11], including SA-AKI, [10, 12-15] by modulating the balance between pro-inflammatory and anti-inflammatory states.

A recent trial in healthy adults confirmed that preventative intravenous (i.v.) treatment with $4 \times 10^{6} \mathrm{MSC} / \mathrm{kg}$ produces early immunomodulatory effects on the host response to LPS [16]. Furthermore, administration of allogeneic MSC to patients with septic shock (NCT01849237, NCT02421484, and NCT02328612) and sepsis-related acute respiratory distress syndrome (NCT01775774) demonstrated good safety and tolerability in Phase 1 clinical trials [16-19]. Despite these encouraging results, Galstyan et al. also reported that a single dose of i.v. MSC did not prevent death from sepsis-related organ dysfunction, raising the possibility that additional doses may be necessary to derive meaningful clinical benefit [17]. A recent non-sepsis, phase 2 trial (NCT01602328) in which single doses of allogeneic MSC were delivered intra-aortically to patients with sterile AKI following cardiopulmonary by-pass surgery also failed to demonstrate a beneficial effect on organ dysfunction and patient survival despite promising pre-clinical and phase 1 trial results [20]. Whether repeated dosing during the early course of sepsis and other acute inflammatory syndromes could augment or extend the disease-modulating effects of

MSC remains relatively under-investigated at pre-clinical and translational levels. Furthermore, incomplete knowledge of the mechanism of action, dose response and optimal clinical indices for MSC administration in sepsis limits the potential for designing successful trials [5, 21, 22].

In the current study, we performed pre-clinical investigation of the anti-inflammatory effects of CD362-selected human umbilical cord-derived MSC (hUC-MSC) in mouse models of sepsis. This surface marker-selected hUC-MSC is a novel therapeutic product that has demonstrated evidence of efficacy in rat models of bacterial pneumonia and sepsis when administered early after disease onset [23, 24]. As a clinical-grade investigational medicinal product (IMP), hUC-MSC is currently undergoing phase I/II trial in patients with moderate to severe acute respiratory distress syndrome (ARDS) due to COVID-19 (NCT03042143) and in multiple autoimmune inflammatory diseases (POLARISE). We aimed to demonstrate the anti-inflammatory potential of single doses of hUC-MSC in mouse models of LPS- and CLPinduced endotoxemia/polymicrobial sepsis $[25,26]$ and, in the latter, to determine whether a second administration of hUC-MSC during the early disease course resulted in greater or more frequent beneficial effects on systemic inflammation and organ-specific injury, exemplified by SA-AKI.

\section{Materials \& Methods}

Cells Anti-CD362 ${ }^{+}$-selected hUC-MSC were cultured from ethically-sourced human umbilical cord tissue obtained from Tissue solutions Ltd. (Glasgow, U.K). Primary isolation and expansion cultures of CD362 ${ }^{+}$hUC-MSC was carried out as previously described $[19,23,27]$. Cryopreserved vials $\left(1 \times 10^{7}\right.$ in $\left.1 \mathrm{~mL}\right)$ of anti-CD362 ${ }^{+}$-selected hUC-MSC were thawed, transferred into $9 \mathrm{~mL}$ of phosphate buffered saline (PBS). After live/dead analysis either via trypan blue dye or automated cell counter (NucleoCounter ${ }^{\circledR} \mathrm{NC}-200^{\mathrm{TM}}$, Chemometec A/S, Denmark), the required numbers of cells were pelleted via centrifugation at $400 \times \mathrm{g}$ for $5 \mathrm{~min}$ and the cells were re-suspended in $100 \mu \mathrm{l}$ of sterile saline for intraperitoneal (i.p.) or i.v. injection.

Animal Procedures All animal procedures were carried out under a license (no. 255/17) from the Animal Experimentation Ethical Committee, University of Barcelona and under authorisation (AE19125/P082 and AE19125/P066) from the Health Products Regulatory Authority, Ireland, and approved by the NUI Galway Animal Care Research Ethics Committee. All procedures were performed in licensed animal facilities at NUI Galway and University of Barcelona.

For the LPS model of endotoxemia, 10-12 week-old male C57BL/6 mice from Charles River Ltd., Kent, UK were used. Mice were injected i.p. with $5 \mu \mathrm{g} / \mathrm{g}$ LPS (LPS 
0111:B4, catalogue no. L2630, Sigma Aldrich, UK) in $100 \mu \mathrm{l}$ of sterile saline. The mice were housed in groups of 3-5 mice/cage during the study in individually ventilated cages. Single i.p. injections of $2.5 \times 10^{5} \mathrm{hUC}-\mathrm{MSC}$ or equal volumes of vehicle (sterile saline) were administered $4 \mathrm{~h}$ after LPS injections. The animals were monitored every $4 \mathrm{~h}$ until the end of the study using a distress score sheet and support measures according to a pre-determined protocol. Humane euthanasia was performed at the defined experimental end-point or earlier if animals exceeded the pre-defined severity score threshold. At the time of euthanasia, peritoneal exudates were collected for flow cytometry analysis by carefully flushing $5 \mathrm{~mL}$ of sterile PBS into and out of the peritoneal cavity.

Cecal ligation and puncture (CLP) was performed on 8-12 week-old, male C57BL/6 mice (Charles River Ltd., UK). The mice received buprenorphine $0.1 \mathrm{mg} / \mathrm{kg}$ (Richter Pharma AG, Austria) subcutaneously 25-30 min before the procedure and were anesthetized with $1.8-2 \%$ isoflurane (with $\mathrm{O}_{2}$ flow of $0.5 \mathrm{~L} / \mathrm{min}$ ) at NUI Galway or with Anesketin $(100 \mathrm{mg} / \mathrm{mL}$; Dechra Veterinary Products SLU, Spain) and Rampun $(20 \mathrm{mg} / \mathrm{mL}$; Bayer, Germany) at University of Barcelona. The lower half of the abdomen was shaved and cleaned with $4 \%$ chlorhexidine or povidone-Iodine and incised $1 \mathrm{~cm}$ vertically along the midline. The cecum was externalized and the distal $50 \%$ was ligated using 4.06.0 M sutures. Cecal material was released by 'through and through' puncture with a 21-gauge needle and a drop of fecal matter was exuded before reinstating the cecum into the peritoneal cavity and suturing the muscle and skin closed. Sham-operated mice underwent an identical procedure, including opening the peritoneum and exposing the bowel, but without ligation and perforation of the cecum. Mice received $0.5 \mathrm{~mL}$ of Gelofusine (Braun Melsungen AG, Germany) by i.p. instillation prior to wound closure. Postoperative support consisted of buprenorphine diluted given subcutaneously (s.c.) every 8-12 h until the pre-determined end-points ( 48 or $72 \mathrm{~h}$ for individual experiments). Administration of $1 \times 10^{6} \mathrm{hUC}-\mathrm{MSC}$ or equivalent volumes of vehicle (sterile saline) was carried out i.v. via the tail vein at $4 \mathrm{~h}$ or at 4 and $28 \mathrm{~h}$ following CLP. Frequent monitoring and support was carried out according to an ethically-approved protocol. Humane euthanasia was performed at the defined experimental end-point or earlier if animals exceeded a predefined severity score threshold.

The cell doses for the two animal models employed for the study were selected on the basis of prior reports of human MSC anti-inflammatory effects in similar models $[9,13,14,28,29]$. The group sizes for the LPS study were selected empirically based on relevant prior reports for this model [30, 31]. The group sizes for the CLP study were determined for a primary outcome of plasma NGAL at $24 \mathrm{~h}$ post-surgery. Using data from a pilot experiment, a sample size of 9 animals per group was calculated to provide $90 \%$ power assuming a 5\% significance level and a two-sided test (http://www.3rs-reduction.co.uk/html/6__power_and_sample_size.html). An expected attrition rate of $10 \%$ was applied to select the final group size of $n=10$.

Blood Sampling and Tissue Procurement Venous blood samples to a maximum volume of $20 \mu \mathrm{L}$ were drawn intermittently from tail and facial veins by aseptic technique using 25-21 gauge needles and were collected into heparin (VWR International, Dublin, Ireland)-containing tubes. A terminal blood sample was drawn by cardiac puncture at the time of euthanasia. Serum was collected in micro-tubes with serum gel and clotting activator (Sarstedt, Wexford, Ireland). Plasma and serum samples were prepared by centrifugation at $10,000 \times \mathrm{g}$ for $10 \mathrm{~min}$. Serum samples were stored at $-80{ }^{\circ} \mathrm{C}$ and subsequently analysed for biochemical parameters by NationWide Laboratories (Lancashire, UK). Spleen, lungs, kidneys and liver were dissected immediately after euthanasia.

Multicolour Flow Cytometry For flow cytometry of mouse peritoneal macrophages (LPS model), $100 \mu \mathrm{L}$ of freshlyprepared peritoneal exudate cells re-suspended in FACS buffer (PBS, $2 \%$ fetal calf serum and $0.05 \%$ sodium azide) were labelled with the following combinations of fluorochrome-labelled antibodies at $4{ }^{\circ} \mathrm{C}$ for $30 \mathrm{~min}$ : anti-Ly6CPerCP-Cy ${ }^{\mathrm{TM}} 5.5$ (clone-AL-21), anti-CD11b-APC-Cy7 (clone-M1/70), anti-CD68-PE (F1/11), anti-CD206-APC (MR5D3) from BD Pharmingen ${ }^{\mathrm{TM}}$ (BD Bioscience, Berkshire, UK). Cell viability was analysed by SYTOX ${ }^{\mathrm{TM}}$ blue dead cell stain (Thermo-Fisher Scientific, Dublin, Ireland) according to manufacturer's instructions. For flow cytometry of kidney cells, single-cell suspensions were prepared from freshly-dissected kidneys by collagenase/DNase digestion and were enriched for $\mathrm{CD} 45^{+}$bone marrow-derived cells by magnetic column separation. Single-cell suspensions of lung were also prepared by collagenase/DNase digestion and mechanical disruption respectively without subsequent CD45-enrichment. Single cell suspensions of spleen were prepared by mechanical disruption. Kidney cell suspensions were stained with panels of fluorochrome-coupled monoclonal antibodies. A detailed protocol for preparation and flow cytometry analysis of cell suspensions is available in Supplementary Methods. For all flow cytometry analyses, labelled cells were washed and re-suspended in FACS buffer and immediately analysed on a FACS Canto II cytometer (BD Biosciences). Data files were subsequently analyzed using FlowJo v6 software (Ashland, OR, USA). Details and examples of the gating strategies used to define and enumerate specific immune cell subpopulations are provided in Supplementary Table 1 and Supplementary Fig. 1. 
A

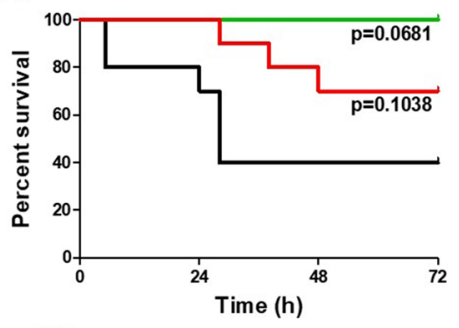

Saline+Saline

LPS+Saline

LPS+0.25M UC-MSC
B

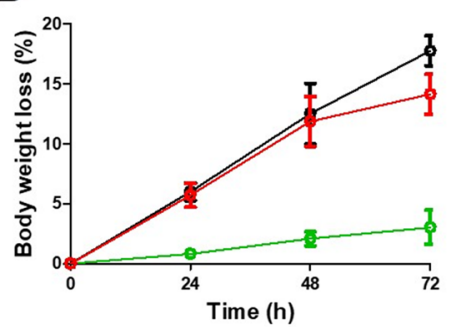

C

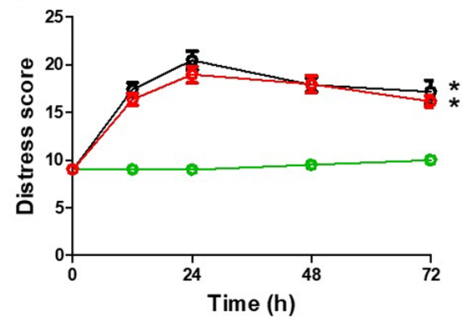

D
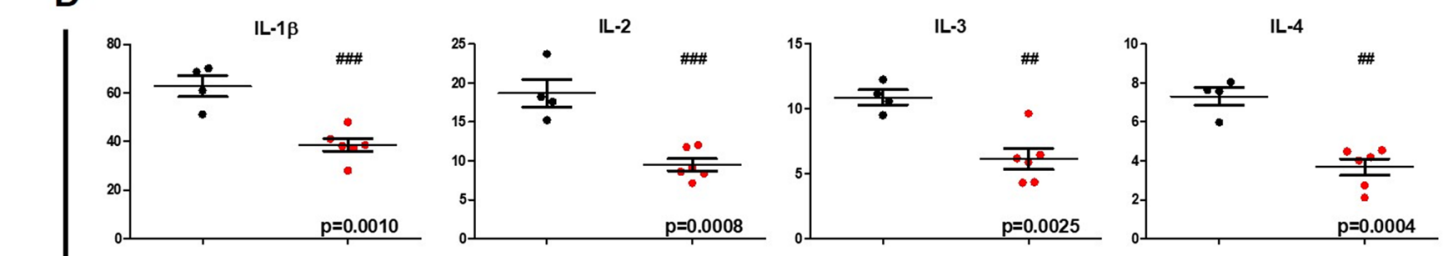

통
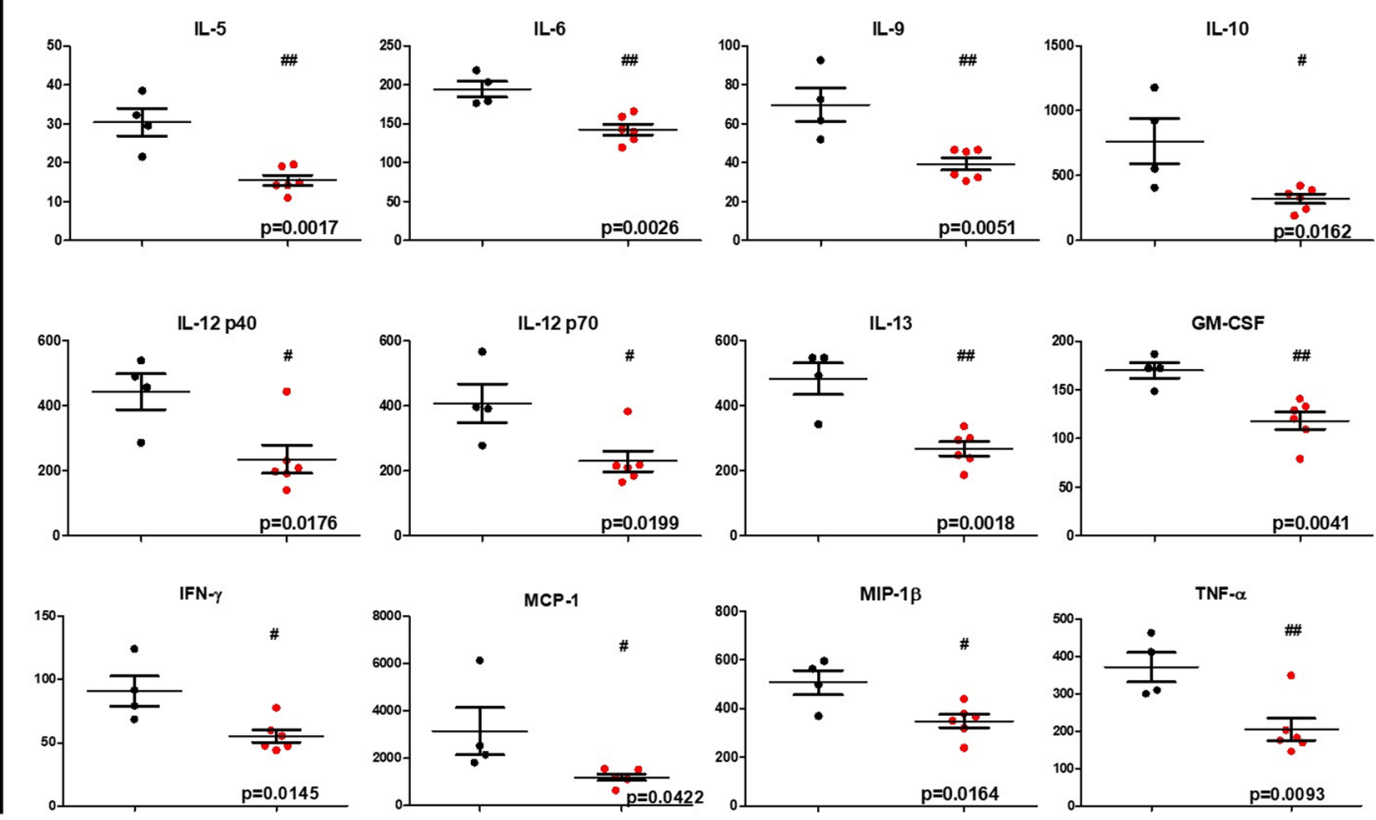

LPS+Saline

LPS+0.25M UC-MSC

E
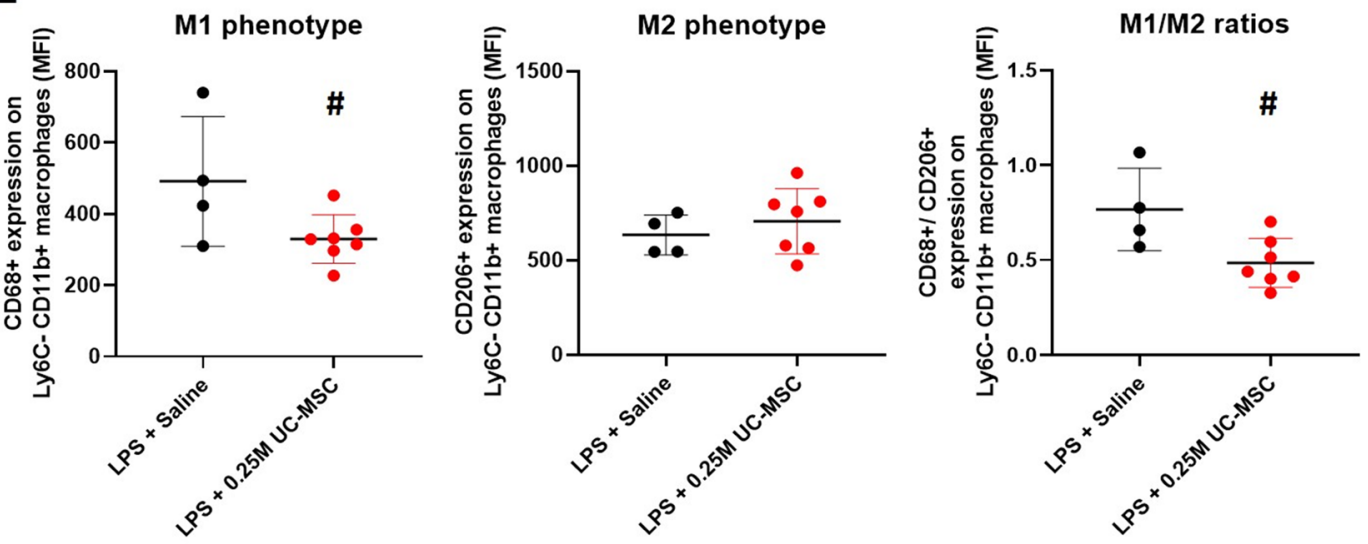
४Fig. 1 Effects of single-dose intra-peritoneal hUC-MSC administration on survival, systemic inflammatory response and peritoneal macrophage polarization following LPS: a-c Survival curve, percentage of body weight loss data and distress score of groups of mice over $72 \mathrm{~h}$ following intra-peritoneal injection of saline alone (SHAM+Saline $[n=4]$ ), LPS and saline (LPS + Saline $[n=10])$ or LPS and $0.25 \times 10^{6} \mathrm{hUC}-\mathrm{MSC}$ (LPS $+0.25 \mathrm{M}$ UC-MSC $[n=10]$ ). d Serum concentrations of inflammatory cytokines and chemokines in mice surviving to $72 \mathrm{~h}$ following LPS and saline (LPS + Saline $[n=5])$ or LPS and $0.25 \times 10^{6}$ hUC-MSC (LPS $+0.25 \mathrm{M} \mathrm{UC-MSC}$ $[n=5]$ ). e Mean fluorescence intensities (MFI) of $\mathrm{Ly}^{-} \mathrm{C}^{-} / \mathrm{CD} 11 \mathrm{~b}^{+}$ peritoneal macrophages for M1 marker CD68 (left) and M2 marker CD206 (middle) as well as CD68/CD206 ratios for mice surviving to $72 \mathrm{~h}$ following LPS and saline (LPS + Saline $[n=4]$ ) or LPS and $0.25 \times 10^{6}$ hUC-MSC (LPS +0.25 M UC-MSC $\left.[n=5]\right)$. Statistical analysis: A) Long-rank test of Kaplan-Meier survival curve, $p$ values indicate statistical comparison with LPS + Saline group. B) Repeated measures ANOVA with Bonferroni post-test. C) Friedman test with Dunn's post-test, 95\% confidence interval. D) Non-Gaussian distribution: Unpaired T test, $p<0.05$. Gaussian distribution: Non-parametric Mann-Whitney test $p<0.05$. E) Unpaired t-test. *Significantly different from SHAM groups $(p<0.05)$. "Significantly different from LPS + Saline group $\left({ }^{\#} p<0.05,{ }^{\#} p<0.01,{ }^{\# \#} p<0.001\right)$

Immunoassays Plasma neutrophil gelatinase-associated lipocalin (NGAL) concentration was quantified with the mouse Lipocalin-2/NGAL Duo-Set ELISA Development kit (R\&D Systems, Minneapolis, MI, USA) according to the manufacturer's suggested protocol (details in Supplementary Methods). For multiplex quantification of cytokines and chemokines in serum, the Bio-Plex Pro mouse cytokine standard 23-plex assay (Bio-Rad, Accuscience) was used according the manufacturer's instructions. Samples were analysed on a Bioplex 200 multiplex ELISA system (BioRad, Accuscience).

Histology and Immunohistochemistry Kidneys were dissected and placed in $10 \%$ neutral buffered formalin for $24 \mathrm{~h}$ at room temperature before being processed in a Leica Tissue Processor ASP300. Tissues were wax-embedded in a Leica EG1150 wax embedder fitted with EG1130 Cold Plate, and 5- $\mu \mathrm{m}$ sections were cut using a Leica RM12235 microtome. Sections were transferred to Superfrost Plus microscope slides (Fisher Scientific Ireland) and dried overnight at room temperature. Histologic staining of sections for hematoxylin and eosin (H\&E) and periodic acid-Schiff (PAS) were performed using standard protocols (details provided in Supplementary Methods).

For immunohistochemical staining, kidney tissue sections were dewaxed in xylene then hydrated in graded ethanol solutions. Heat-mediated antigen retrieval was performed with Tris/EDTA buffer $\mathrm{pH} 9.0$ at $90{ }^{\circ} \mathrm{C}$ for $30 \mathrm{~min}$. Sections were treated with $0.3 \% \mathrm{H}_{2} \mathrm{O}_{2}$, and incubated with avidinbiotin blocking solution (SP-2001, Vector Laboratories, Burlingame, CA, USA) to reduce nonspecific staining. Next, the slides were incubated for one hour at $4{ }^{\circ} \mathrm{C}$ with rabbit anti-mouse NGAL monoclonal antibody (1:2000; ab216462,
Abcam, Cambridge, UK), followed by incubation with biotinylated goat anti-rabbit IgG secondary antibody (BA-1000). For colorization, an avidin-biotin horseradish peroxidase complex (Vector Laboratories) and 3,3-diaminobenzidine substrate solution (Sigma-Aldrich) were applied to the slides at room temperature for 30 and $5 \mathrm{~min}$ respectively and the slides were counterstained with Gill no. 3 hematoxylin (Sigma-Aldrich) for $30 \mathrm{~s}$. Negative control slides were prepared by staining under identical conditions but without adding the primary antibody.

Semi-Quantitative Scoring of Kidney Tissue Sections Stained sections of kidney were analyzed in blinded fashion by light microscopy at 40X magnification using an Olympus BX43 bright-field microscope (Olympus, Center Valley, PA) and with IS TCapture software (Tucsen Photonics Co., Fujian, China). For each kidney, twenty non-overlapping fields of a stained section were captured, and the positively stained area was scored by a blinded observer for (A) tubular dilatation, cast and necrosis (PAS) and (B) NGAL expression [32] Scoring was carried out on a 0-4 semi-quantitative scoring scale (details in Supplementary Methods). Mean scores were calculated for each individual kidney, and final results were expressed as group means $\pm \mathrm{SD}$.

Statistical Analysis Results were expressed as means \pm $\mathrm{SD}$, and differences between conditions were tested statistically by ANOVA and post-hoc tests where indicated using GraphPad Prism 5 software (GraphPad Software, La Jolla, CA). Significance was assigned at $p<0.05$. Unsupervised hierarchical clustering and the generation of the corresponding heat map were performed using the Morpheus online visualization and analysis suite (Broad Institute, Cambridge, MA, https://software.broadinstitute.org/morpheus). All data, except gamma glutamyl-transpeptidase (Gamma GT) and albumin-globulin ratio (Albumin:Globulin), were subjected to $\log 2$ transformation in Excel (version 2013, Microsoft, Redmond, WA), prior to clustering. The hierarchical relationship of data patterns for all animals was establish by average linkage, Euclidean distance method.

\section{Results}

A Single Dose of hUC-MSC Modulated Inflammatory Response to LPS in Mice To initially establish a modulatory effect of hUC-MSC on acute systemic inflammatory response in mice, the LPS endotoxemia model was used. As shown in Fig. 1a, i.p. administration of $2.5 \times 10^{5}$ hUCMSC $4 \mathrm{~h}$ after LPS injection was associated with higher 72-h survival compared to a control group that received LPS followed by i.p. saline ( $70 \%$ vs. $40 \%$ survival). However, the difference did not reach statistical significance and the 
A
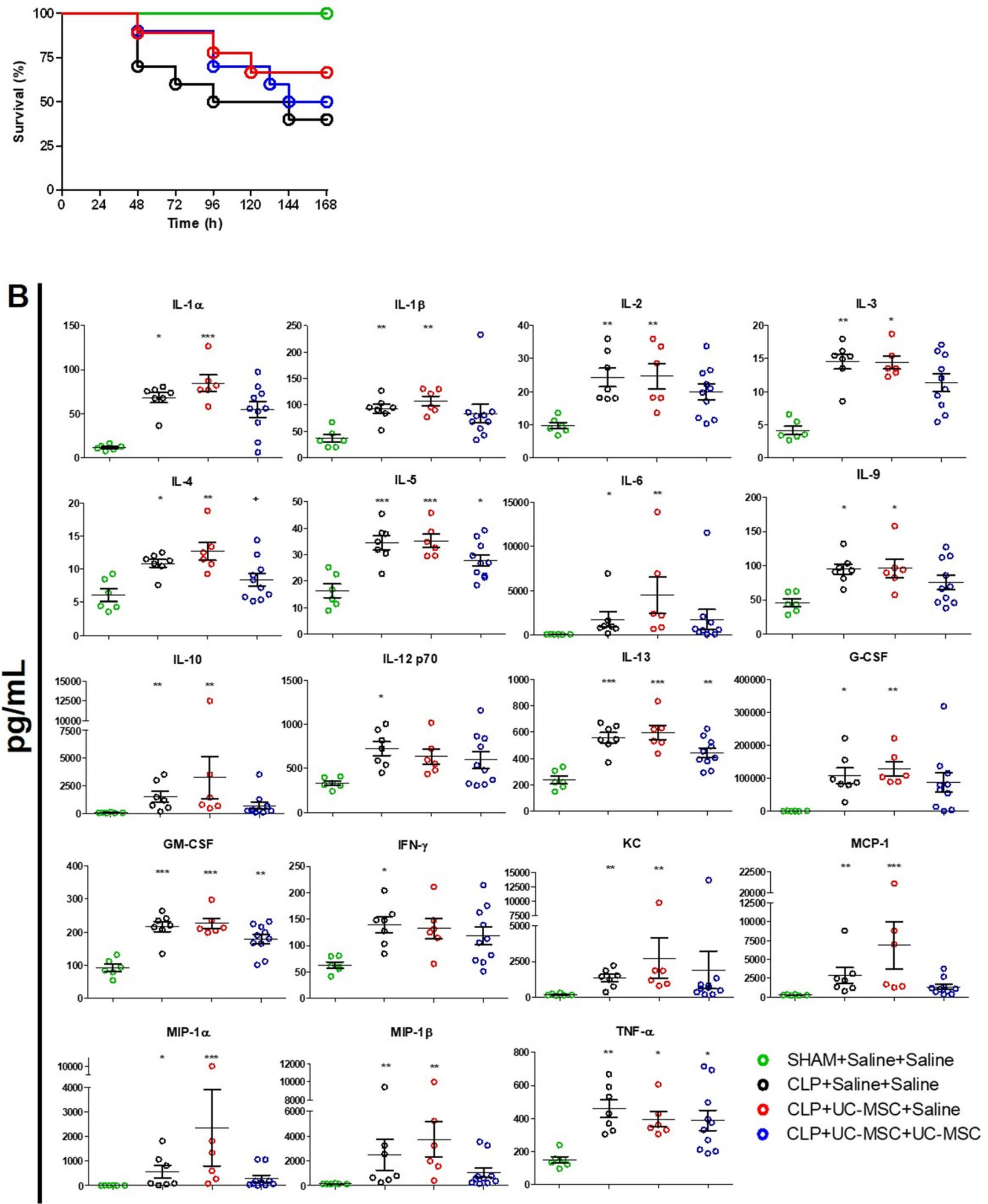
4 Fig. 2 Effects of single- and double-dose intravenous hUC-MSC on survival and systemic inflammatory responses following CLPinduced polymicrobial sepsis: a Survival curves for groups of mice following sham procedure with saline injections at 4 and $28 \mathrm{~h}$ (SHAM+Saline+Saline, $[n=5])$, CLP procedure with saline injections at 4 and $28 \mathrm{~h}(\mathrm{CLP}+$ Saline+Saline, $[n=10])$, CLP procedure with $1 \times 10^{6} \mathrm{hUC}-\mathrm{MSC}$ at $4 \mathrm{~h}$ and saline injection at $28 \mathrm{~h}$ (CLP+UCMSC + Saline, $[n=9])$ or CLP procedure with $1 \times 10^{6}$ hUC-MSC at $4 \mathrm{~h}$ and at $28 \mathrm{~h}(\mathrm{CLP}+\mathrm{UC}-\mathrm{MSC}+\mathrm{UC}-\mathrm{MSC},[n=9])$. Animals were monitored for $168 \mathrm{~h}$ following procedures. b Serum concentrations of inflammatory cytokines and chemokines at $48 \mathrm{~h}$ following the same combinations of procedures and treatments. Groups sizes for the multiplex assay analyses were SHAM+Saline+Saline $(n=6)$, CLP + Saline+Saline $(n=7)$ and CLP + UC-MSC + Saline $(n=6)$ or CLP + UC-MSC + UC-MSC $(n=10)$. Statistical analysis: a Long-rank test of Kaplan Meier survival curve b Non-Gaussian distribution: Kruskal-Wallis test with Dunn's multiple comparison test, $p<0.05$. Gaussian distribution: One-way ANOVA with Bonferroni multiple comparison post-test and with $95 \%$ confidence interval. *Significantly different from SHAM group $\left({ }^{*} p<0.05\right.$, $* * p<0.01$, $* * * p<0.001)$

average body weight losses and total distress scores over $72 \mathrm{~h}$ were closely comparable for the two groups (Fig. 1b and c). Despite the limited effects on physical determinants of acute illness, multiplexed analysis of soluble inflammatory mediators in serum of animals that survived to $72 \mathrm{~h}$ revealed significant reductions in hUC-MSC recipients in comparison to the saline group (Fig. 1d). Notably, circulating concentrations of key mediators of innate (e.g. IL-1 $\beta$, IL-6, GM-CSF, TNF $\alpha$, IL-10, MCP-1, MIP-1 $\beta$ ) and Th1and Th2-type adaptive immune responses (e.g. IL-2, IFN $\gamma$, IL-12, IL-3, IL-4, IL-5, IL-13, IL-9) were lower in hUCMSC-treated compared to saline-treated animals. Concentrations of the Th17-type mediator IL-17, the eosinophil chemoattractant, eotaxin and the chemokine CCL5 were unaffected by hUC-MSC administration (data not shown). To assess the immunomodulatory effects of hUC-MSC at the site of delivery, $\mathrm{CD} 11 \mathrm{~b}^{+} / \mathrm{Ly} 6 \mathrm{C}^{-}$peritoneal macrophages from surviving animals were analysed by flow cytometry for surface expression levels of the M1 and M2 markers CD68 and CD206. As shown in Fig. 1e, peritoneal macrophages from hUC-MSC-treated animals had lower CD68 surface expression and lower CD68/CD206 ratio compared to those from saline-treated animals. Overall, these results demonstrated that a single dose of hUC-MSC had distinct anti-inflammatory and immune-modulatory effects in a simplified sepsis-like model with limited influence on overall disease severity.

\section{Repeated Doses of hUC-MSC Modified Survival and Systemic Inflammation Following CLP-Induced Polymicrobial Sepsis} in Mice To assess (i) whether the anti-inflammatory effects of hUC-MSC could be reproduced in a more clinically-relevant model and route of administration and (ii) whether a repeat dose of cells early in the disease course could more potently modulate the course of sepsis, experiments were subsequently performed in the mouse CLP model. Groups of animals underwent CLP or sham procedures and received i.v. injections of $1 \times 10^{6}$ hUC-MSC or equivalent volumes of saline at $4 \mathrm{~h}$ only or at 4 and $28 \mathrm{~h}$ after CLP. In the first such experiment, survival over a prolonged period (7 days) was determined (Fig. 2a). As shown, CLP animals administered saline alone at both time points had 40\% 7-day survival compared to $100 \%$ for sham controls. Seven-day survival of groups that received hUC-MSC at $4 \mathrm{~h}$ only (single dose) or at 4 and $28 \mathrm{~h}$ (double dose) did not differ significantly from that of the untreated group. It was noted, however, that mortality of animals in both hUC-MSC groups tended to occur later than that of untreated CLP animals. To explore this further, a second study with a duration of $48 \mathrm{~h}$ was performed using the same groupings and group sizes. As shown in Table 1, when the 48-h survivals of the combined total animals for each of the 4 groups from the 2 experiments were compared, those that received two doses of hUC-MSC had $95 \%$ survival compared to $75 \%$ and $74 \%$ respectively for untreated and single dose-treated groups. This difference in survival did not reach statistical significance.

To investigate further, serum samples collected from surviving animals at the end-point ( $48 \mathrm{~h}$ post-CLP) of the second experiment were analyzed by multiplex assay for soluble mediators of innate and adaptive immune responses (Fig. 2b). As shown, serum concentrations of the majority of these cytokines and chemokines were significantly elevated in untreated CLP compared to sham animals. As with the LPS model, serum concentrations of IL-17, eotaxin and CCL5 were unaffected by CLP (data not shown). With the exception of IL-12p70 and IFN $\gamma$, all CLP-induced mediators were also significantly elevated in the single-dose hUCMSC-treated group. For the double-dose hUC-MSC group, however, multiple innate immune mediators (IL- $1 \alpha$, IL-1 $\beta$, IL-6, IL-10, G-CSF, KC, MCP-1, MIP-1 $\alpha$, MIP-1 $\beta$ ) and some Th1 - and Th2-type adaptive immune mediators (IFN $\gamma$, IL-2, IL-3, IL-9) did not differ significantly from the sham group. The findings supported a conclusion that repeated dosing of hUC-MSC is necessary for detectable modulation of systemic inflammation $48 \mathrm{~h}$ after onset of polymicrobial sepsis.

The CLP Model Was Not Associated with Multi-Organ Failure To determine whether the CLP model was associated with multi-organ failure that may have been modulated by single- and/or double-dose hUC-MSC administration, biochemical analyses reflecting liver, kidney and pancreas function were performed on 48-h serum samples collected at the termination of the second experiment (Fig. 3). As shown, there were trends for individual group results consistent with adverse changes in liver and pancreas function tests (AST, albumin, Amylase, ALT, GGT) in CLP compared to sham 
Table 1 Survival to $48 \mathrm{~h}$ following CLP in combined groups from two experiments comparing effects of saline, single-dose hUC-MSC and double-dose hUC-MSC

\begin{tabular}{|c|c|c|c|c|c|c|}
\hline \multirow[b]{2}{*}{$\begin{array}{l}\text { Experimental Group } \\
\text { (Procedure/IV inject. at } 4+28 \text { h) }\end{array}$} & \multicolumn{5}{|c|}{ Time-point (No. of animals surviving) } & \multirow[b]{2}{*}{$\begin{array}{l}\% \text { Sur- } \\
\text { vival to } \\
48 \mathrm{~h}\end{array}$} \\
\hline & $0 \mathrm{~h}$ & $12 \mathrm{~h}$ & $24 \mathrm{~h}$ & $36 \mathrm{~h}$ & $48 \mathrm{~h}$ & \\
\hline SHAM / Saline + Saline & 14 & 14 & 14 & 14 & 14 & 100 \\
\hline CLP / Saline + Saline & 20 & 20 & 20 & 20 & 15 & 75 \\
\hline CLP / hUC-MSC + Saline (single-dose) & 19 & 19 & 19 & 19 & 14 & 74 \\
\hline CLP / hUC-MSC + hUC-MSC (Double-dose) & 20 & 20 & 20 & 20 & 19 & 95 \\
\hline
\end{tabular}

groups. In contrast, apart from a mild increase in urea in one group, serum markers of kidney function (creatinine, urea, potassium) were not different among the groups. These results indicated that, while the CLP model we established resulted in systemic inflammation and reduced survival, multi-organ failure was not present at the time and antiinflammatory effects of repeated hUC-MSC administration were observed.

\section{Repeated Dosing of hUC-MSC Modulated the Severity of} Acute Kidney Injury Following CLP-Induced Polymicrobial Sepsis in Mice To more clearly determine whether the antiinflammatory effect of repeated hUC-MSC administration was associated with end-organ protection, we focussed on the kidneys. Additional analyses of AKI were performed on 48-h samples from each group of the second CLP experiment. Neutrophil gelatinase-associated lipocalin-2 is a biomarker that increases rapidly in blood and urine following the onset of sterile AKI as well as SA-AKI [33, 34]. As shown in Fig. 4a, plasma NGAL concentrations were markedly increased in CLP compared to sham control animals at $48 \mathrm{~h}$. In the double-, but not the single-dose, hUC-MSC group, however, the average plasma NGAL of animals surviving to $48 \mathrm{~h}$ was lower than that of the untreated CLP group. At the same time-point, the intensity of renal tubular staining for NGAL, determined by immunohistochemical staining of kidney tissue sections, was also markedly increased in CLP compared to sham controls (Fig. 4b and e). Although not reaching statistical significance, there was a trend towards lower renal tubular NGAL staining among the double-dose hUC-MSC group compared to untreated and single-dose hUC-MSC groups. To support the conclusion that plasma NGAL reflected severity of AKI rather than being a non-specific marker of sepsis-associated inflammation, there was a significant correlation between plasma NGAL and renal tubular NGAL staining intensity at $48 \mathrm{~h}$ among all CLP animals (Fig. 4c). To determine whether overt tubular necrosis and/or inflammatory renal injury was present, semi-quantitative scoring of PAS-stained tissue sections for tubular damage and interstitial cell infiltrates was carried out. This analysis failed to demonstrate clear differences among the sham and CLP groups (Fig. 4d and e). Furthermore, a blinded analysis carried out by a consultant histopathologist $(\mathrm{SOH})$ for features of ischemia (necrosed tubules, loss of $\mathrm{PAS}^{+}$brush border, cell swelling and oedema, the presence of protein casts, neutrophils and capillary collapse) indicated only mild, variable abnormalities that did not differ between Sham and CLP groups or among the three CLP groups (data not shown).

In kidney cell suspensions from the same experimental groups, magnetic enrichment and multi-colour flow cytometry were used to quantify total $\left(\mathrm{CD} 45^{+}\right)$intra-renal immune cells as well as several myeloid and lymphoid immune cell subpopulations (Fig. 5). As shown, kidneys of the surviving animals from the untreated and single-dose hUC-MSC CLP groups demonstrated reduced numbers of total immune cells and of all myeloid and lymphoid subpopulations compared to the sham controls. For the double-dose hUC-MSC-group, however, intra-renal numbers of total immune cells as well as of $\mathrm{CD}^{+}{ }^{+} \mathrm{T}$-cells, $\mathrm{CD}^{-} / \mathrm{CD}^{-}$(double negative) T-cells, neutrophils and $\mathrm{Ly} 6 \mathrm{C}^{-} / \mathrm{F} 4 / 80^{+}$macrophages did not differ significantly from those of sham controls. These observations were consistent with sepsis-associated depletion of intra-renal myeloid and lymphoid immune cell populations at the time-point studied that was partially reversed by double- but not single-dose i.v. hUC-MSC. Analysis of dead/ dying cells among the renal $\mathrm{CD}_{4} 5^{+}$cells for each group (based on positive staining for the viability dye eFluor 506) revealed reduced proportions of live cells in samples from untreated and single-dose hUC-MSC groups compared to sham controls but not in those from the double-dose group (Supplementary Fig. S2). Overall, the results for analyses focussed on the kidneys at $48 \mathrm{~h}$ after onset of polymicrobial sepsis, suggested that, despite the inconsistent rise in urea/ creatinine and lack of overt acute tubular necrosis, there was a substantial acute injury response and increased intrarenal immune cell death. Furthermore, repeated i.v. dosing of hUC-MSC was associated with evidence of amelioration of these abnormalities.

In keeping with a sepsis-associated immune depletion that also impacted other organs and tissues, flow cytometric analysis of cell suspensions from lungs and spleens of the same animals at $48 \mathrm{~h}$ post-CLP also demonstrated reduced total numbers of CD45 ${ }^{+}$cells and of some lymphoid and 
myeloid subpopulations, that was less marked in the doubledose treated group (Supplementary Figs. S3 and S4).

\section{Cluster Analysis of Multiple Blood Analytes Reveals Response Trends and Intra-Group Heterogeneity Following Repeated Dosing of hUC-MSC in CLP In keeping with the complex} nature of polymicrobial sepsis, many of the analyses shown in Figs. 2, 3, 4 and 5 for untreated, single- and double-dose hUC-MSC-treated CLP groups were associated with high intra-group variability and with trends toward beneficial effects of repeated dosing or non-significant differences to the sham control group. To better understand whether these results represented evidence of a specific hUC-MSCassociated "responder profile" among that experimental group, an unsupervised hierarchical clustering analysis was performed of all factors quantified in blood of surviving animals at the 48-h time-point after initiation of CLP (Fig. 6). As shown, the cluster containing all 5 of the sham group animals was divided from the rest of the animals by the greatest Euclidean distance and this same cluster also included 2/10 double-dose i.v. hUC-MSC-treated CLP animals. Other clusters contained mixed populations from each of the 3 CLP groups. Overall, while suggesting that animals treated with two sequential i.v. doses of hUC-MSC were more likely than those from other groups to resemble nonseptic animals, this analysis highlighted the wide variability in systemic markers of sepsis for the CLP model and did not indicate a distinct signature of disease modulation following hUC-MSC administration.

\section{Discussion}

Sepsis and its frequent complication SA-AKI are major public health challenges due to the continued lack of effective treatments and disappointing results from late-stage clinical trials [2,3]. Several recent pre-clinical studies have reported results indicating that MSC of various sources and their products have positive effects on disease severity and survival in models of sepsis and AKI [7, 8, 11-15]. Nonetheless, the clinical benefits of MSC in sepsis and SA-AKI remain unproven with only limited data available from human patients [5]. To date, clinical trials have documented that single-dose i.v. MSC infusion in the setting of LPS administration, sepsis and ARDS is safe and feasible, [16-19] but does not overtly reduce death from sepsis-related organ failure [17]. In this study, we compared the effects of single- and double-dose i.v. administration of a distinctive human MSC therapeutic product (CD362selected hUC-MSC) in the mouse CLP model of polymicrobial sepsis. Initially, in keeping with recently-reported results for this same MSC product in rat models of bacterial pneumonia and sepsis $[23,24]$, we confirmed the potential for early administration of hUC-MSC to modulate LPS-induced systemic inflammation in mice. In a mouse CLP model of polymicrobial sepsis, however, our results indicated that single doses of hUC-MSC administered i.v. $4 \mathrm{~h}$ following induction of fecal peritonitis exerted no clear beneficial effect on systemic inflammation or organ-specific (renal) tissue injury. In contrast, administration of a second i.v. dose of hUC-MSC $24 \mathrm{~h}$ later resulted in multiple signals of ameliorated inflammation and renal injury by $48 \mathrm{~h}$ following onset of sepsis. Despite this, a survival benefit for the double-dose regimen was not definitively demonstrated. Our results highlight the importance of performing in vivo studies of MSC therapeutic products in multiple models and of reporting both positive and negative pre-clinical outcomes in order to better inform clinical translation and trial design. Taken together, our results also provide evidence that the limited or absent benefit of early, single-dose regimens of anti-inflammatory MSC products in sepsis trials may be at least partially overcome by repeated dosing.

In the mouse LPS model, a single, early dose of hUCMSC modulated systemic levels of both innate and adaptive inflammatory mediators for at least $72 \mathrm{~h}$, albeit without an overt effect on severity scores and survival. Phenotypic analysis of peritoneal macrophages provided evidence that hUC-MSC administration was associated with a predicted local immune modulatory effect - skewing of macrophages toward M2 polarization, which has been linked to resolution of inflammatory injury and promotion of tissue repair [29, 35] and is likely to be mediated by MSC cross-talk with resident myeloid cells [36]. Indeed, recent studies have reported that therapeutic immunomodulation by MSC in the setting of sepsis may be dependent on their phagocytosis by myeloid cells (mononuclear phagocytes) which then undergo alternative activation resulting in the production of IL-10 and other paracrine anti-inflammatory mediators [7, 27, 37]. Although it would have been of interest to determine whether the benefits of such immunomodulation are enhanced by repeated doses of hUC-MSCs following LPS administration, we reasoned that an experimental model which better reflected an evolving sepsis would have more clinical relevance. Thus, having confirmed hUC-MSC biological activity in mice using LPS administration, the mouse CLP model was used for characterization of anti-inflammatory effects related to repeated dosing and quantification of kidney-specific effects of hUC-MSC in the setting of polymicrobial sepsis and SA-AKI. In our hands, this model was associated with moderately severe sepsis (approximately $70 \%, 50 \%$ and $40 \%$ survival at 48, 96 and $168 \mathrm{~h}$ respectively in untreated animals) without overt liver and kidney failure. Our observations for the model, including mortality rates and trends in serum liver parameters and albumin, are in keeping with the very comprehensive profiling of mouse CLP reported by $\mathrm{Li}$ et al. [38], which also documents reduced body temperature, 

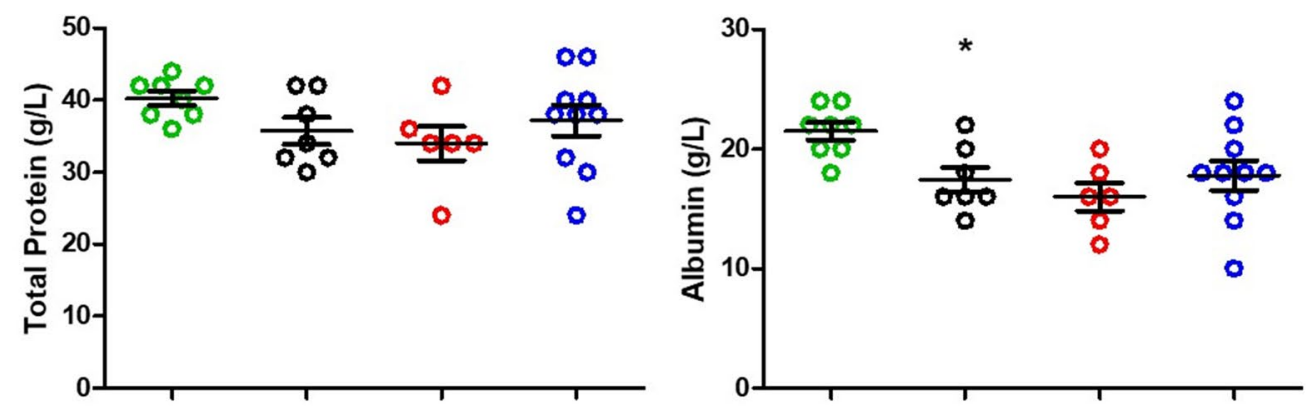

- SHAM+Saline+Saline

- CLP+Saline+Saline

- CLP+UC-MSC+Saline

- $\quad$ CLP+UC-MSC+UC-MSC
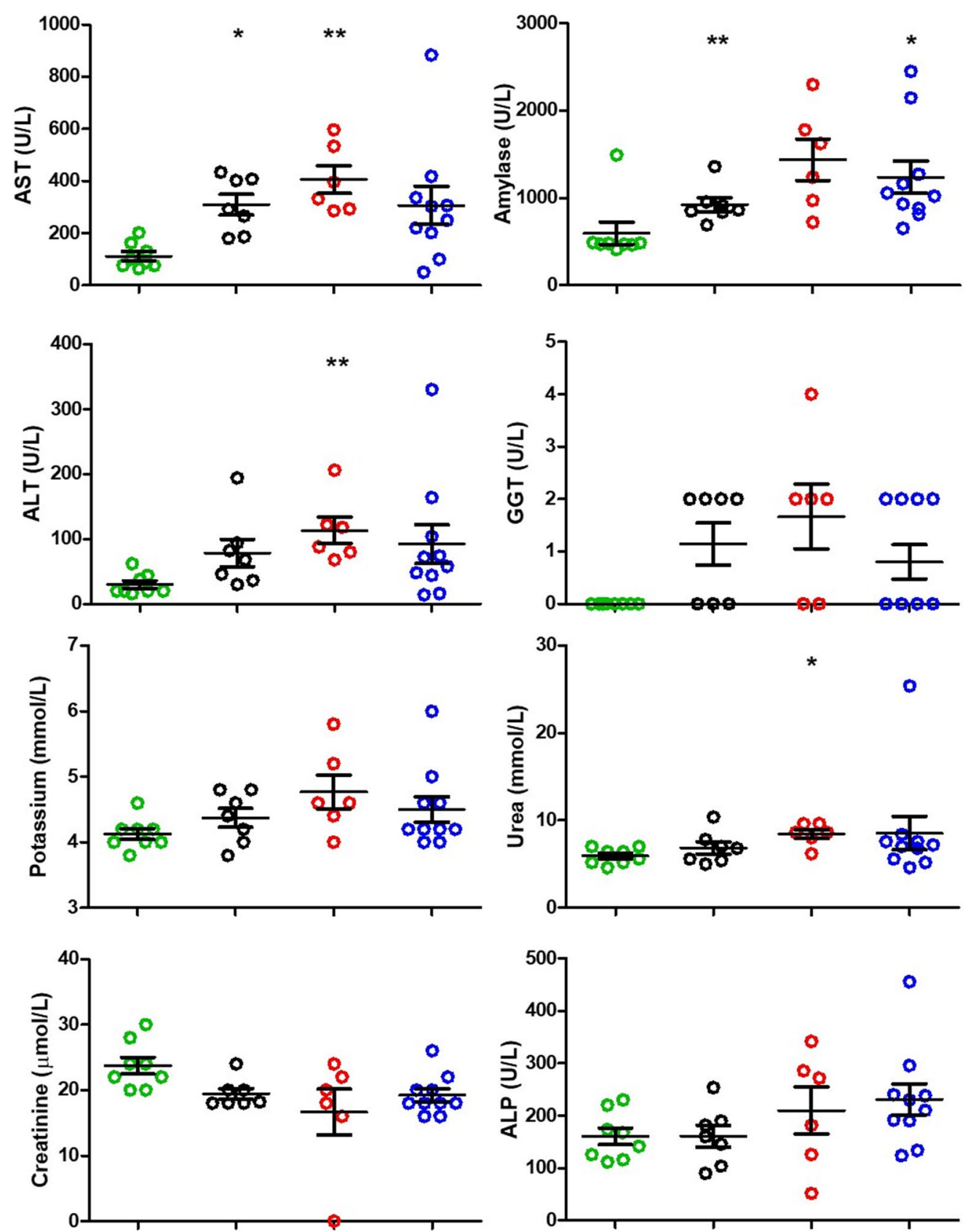
4 Fig. 3 Results of serum biochemistry assays at 48 h following Sham procedure or CLP procedure with injections of saline or hUC-MSC: Results for serum biochemical assays at $48 \mathrm{~h}$ post-procedure are shown for the following groups: sham procedure with saline injections at 4 and $28 \mathrm{~h}($ SHAM+Saline+Saline, $[n=8])$, CLP procedure with saline injections at 4 and $28 \mathrm{~h}(\mathrm{CLP}+$ Saline+Saline, $[n=7])$, CLP procedure with $1 \times 10^{6} \mathrm{hUC}-\mathrm{MSC}$ at $4 \mathrm{~h}$ and saline injection at $28 \mathrm{~h}(\mathrm{CLP}+\mathrm{UC}-\mathrm{MSC}+$ Saline, $[n=6])$ or CLP procedure with $1 \times 10^{6} \mathrm{hUC}-\mathrm{MSC}$ at $4 \mathrm{~h}$ and at $28 \mathrm{~h}$ (CLP + UC-MSC + UC-MSC, $[n=10])$. Statistical analysis: Non-Gaussian distribution: KruskalWallis test with Dunn's multiple comparison test, $p<0.05$. Gaussian distribution: One-way ANOVA with Bonferroni multiple comparison post-test and with $95 \%$ confidence interval. *Significantly different from SHAM group $\left({ }^{*} p<0.05, *^{*} p<0.01\right)$. Abbreviations: AST $=$ Aspartate Aminotransferase $; \quad$ ALT $=$ Alanine Aminotransferase , GGT $=$ Gamma-Glutamyl Transferase $;$ ALP $=$ Alkaline Phosphatase

blood pressure and heart rate during the first $48 \mathrm{~h}$ post-CLP. Interestingly, while $\mathrm{Li}$ et al. documented increased serum creatinine and blood urea nitrogen at 8 and $16 \mathrm{~h}$ post-CLP, their results indicate that these renal functional biomarkers had fallen to normal (or below normal) levels by $48 \mathrm{~h}$ - perhaps reflecting evolving effects of altered metabolism/muscle mass on these biomarkers as the model progresses [38]. Consistent with this, analysis of serum/plasma and kidney tissue at $48 \mathrm{~h}$ indicated significant systemic inflammation and renal injury response without overt evidence of ischemic damage/necrosis. These latter analyses provided the clearest evidence for a modulatory effect of the double-dose hUCMSC regimen on the severity of polymicrobial sepsis when compared to single-dose administration which, in contrast to results recently reported in a rat CLP model [24], was indistinguishable from the saline-treated CLP group across all indices examined. In particular, the clinically-relevant AKI biomarker, NGAL, proved to be a valuable discriminator of sepsis severity and treatment effect in this model and may be an important biomarker for future clinical trials of cell therapies in sepsis and SA-AKI [33, 39].

Our results for the effect of i.v. MSC on survival in small animal models of sepsis are in contrast to some other reports $[28,40]$ but, notably, are in keeping with observed effects of allogeneic MSCs in human clinical trials [5, 16-18, 41]. In this regard, we would highlight the potential role of publication bias - specifically, selective publication of results reflecting positive effects - as an important driver of unrealistic expectations for the efficacy of single-dose MSC regimens and other advanced therapies in the earliest stages of clinical translation [42]. Indeed, Sun et al., in a recent meta-analysis of 29 animal studies of the efficacy of MSC therapies in sepsis, detected significant publication bias and lack of clarity in regard to optimal cell dose among these pre-clinical reports [43]. Nevertheless, we also report distinctive positive findings that may help to advance translational goals for UC-MSCs in sepsis or other systemic and organ-specific inflammatory diseases, such as AKI, liver or respiratory diseases. In both models, these molecular changes indicate complex interactions of hUC-MSC with the Th1 and Th2 immune response. Such effects on T-effector cell activation and $\mathrm{T}$ helper phenotype balance may play a key role in modulation of the acute phase of sepsis as indiscriminate, dysregulated activation of immune effectors resulting in high levels of circulating cytokines contribute to multi-organ failure [44] and, in the case of T-cells, may be followed by widespread apoptosis and subsequent immune deficiencies [45]. It has also been shown that MSC administration may decrease localized tissue inflammation by regulating cytokine homeostasis and decreasing the traffic of immune cells into organs [44]. In keeping with this, our quantitative analysis of a range of intra-renal immune cell populations $48 \mathrm{~h}$ following CLP-indued sepsis revealed sepsis-induced deficiencies affecting both innate and adaptive effectors, including loss of double-negative $\mathrm{T}$ cells which have been recently reported to be early responders to AKI [46]. Notably, for double- but not single-dose hUC-MSCtreated animals, there was evidence of reversion of intrarenal immune cell depletion. As similar trends were also observed in lungs and spleen, our results indicate the potential for repeated i.v. dosing of MSC to broadly ameliorate immune cell depletion from non-lymphoid and lymphoid organs - a facet of sepsis that has been linked to subsequent mortality due to secondary infection [47, 48]. Based on the additional observation of increased cell death among intrarenal $\mathrm{CD}_{4} 5^{+}$cells in untreated CLP animals, it is plausible that this reflects direct or indirect effects of hUC-MSC to reduce mitochondrial dysfunction and pro-apoptotic signalling[49-51]. Nonetheless, more focussed experiments will be required to fully elucidate the mechanisms by which systemic MSC administration preserves myeloid and/or lymphoid cell numbers in sepsis and to determine whether they can be exploited therapeutically.

Given the inherent variability we and others observe with individual outcomes for the mouse CLP model, we hypothesized that hierarchical clustering analysis of quantitative readouts for a range of circulating inflammation-related mediators at $48 \mathrm{~h}$ would help to better define a distinctive "responder signature" among MSC-treated animals. Although $60 \%$ of the double-dose group clustered in a pattern that separated them from the majority of the single dose- and untreated groups and was closer to the sham group, no very clear multi-analyte profile of disease modulation could be identified. A principal component analysis (PCA) approach yielded a similar conclusion (data not shown). It should be acknowledged that data from animals that failed to survive to $48 \mathrm{~h}$ could not be acquired and it is possible that analyses at one or more earlier time-points could provide a more distinctive responder/non-responder separation. Nonetheless, this analysis highlights the complexity of inter-individual variation that is inherent to animal 


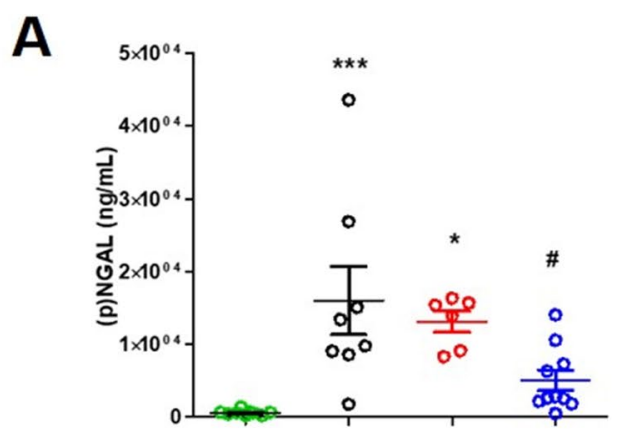

B
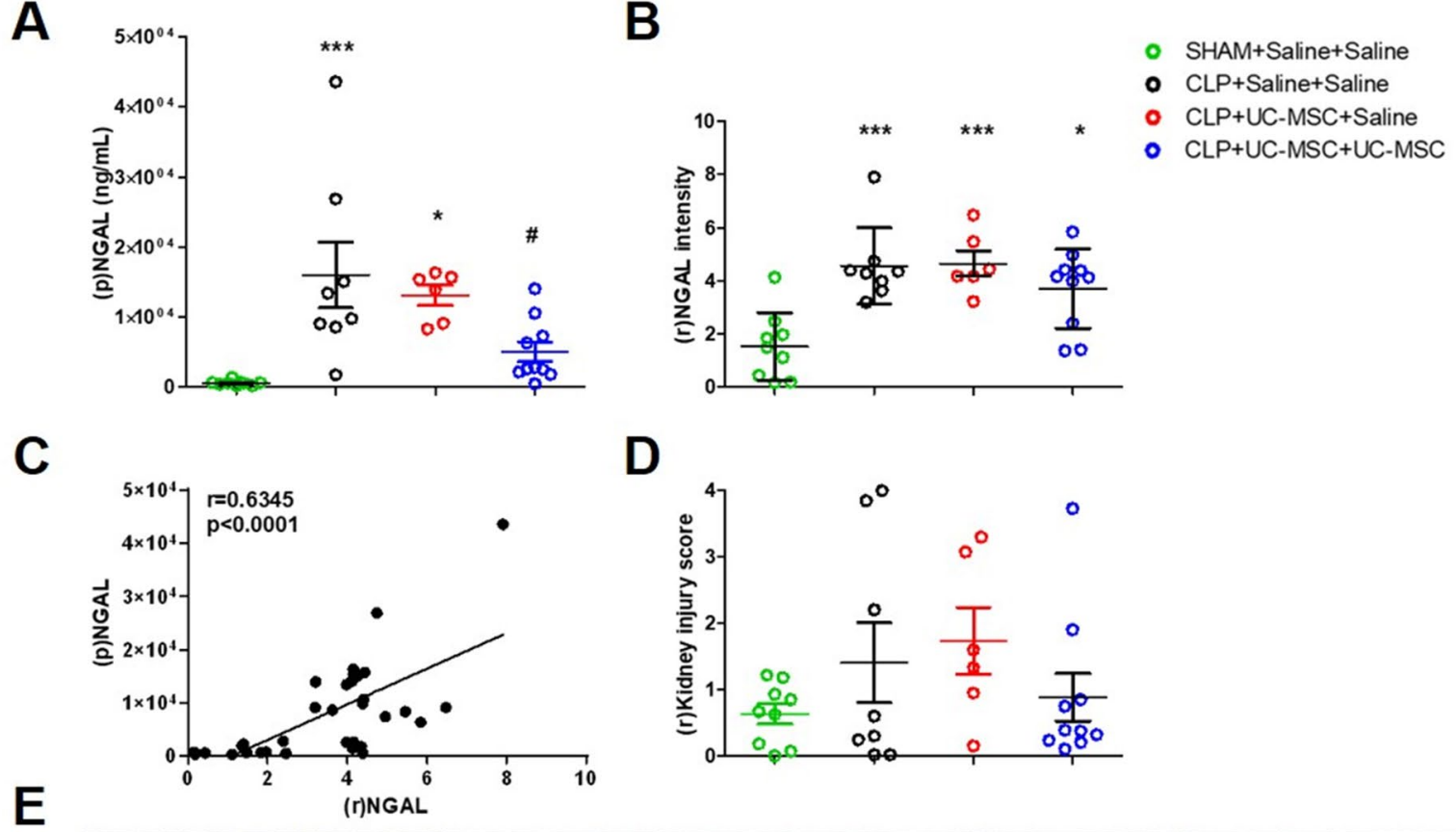

D

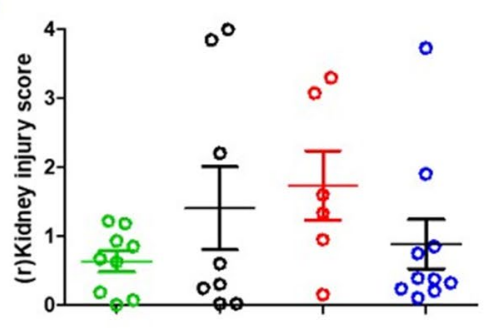

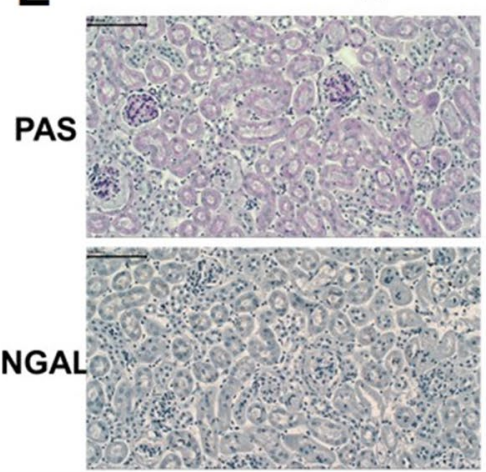

Sham

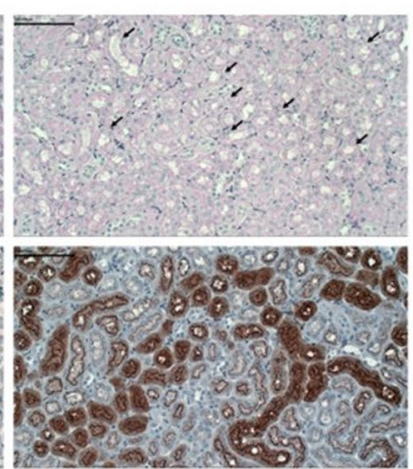

Vehicle

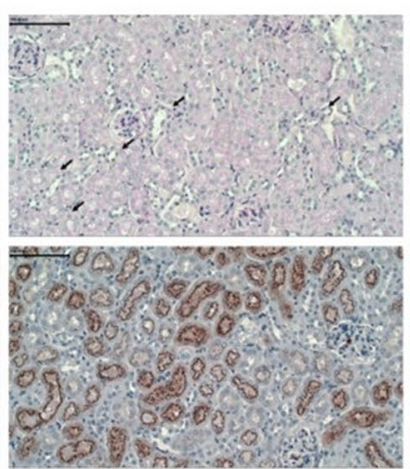

UC-MSC (single)

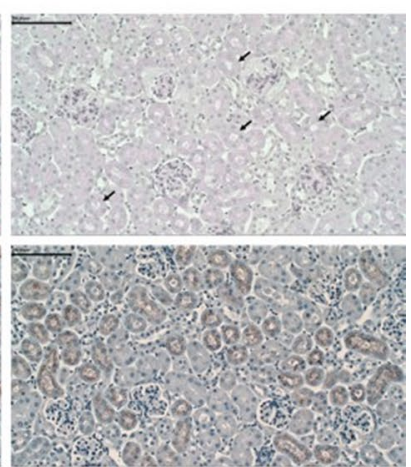

UC-MSC (double)
Fig. 4 Markers of acute kidney injury 48 h following Sham or CLP procedures with saline, single-dose $h U C-M S C$ and double-dose $h U C-M S C$ : a Plasma (p)NGAL concentrations. b Semi-quantitative scoring of renal tubular (r)NGAL staining intensity in kidney sections c Scatter plot illustrating the correlation between 48-h (p) NGAL and (r)NGAL for individual animals from all CLP groups. d Total kidney injury scores, representing the sum of individual semi-quantitative scores for tubular dilatation, cast formation and necrosis in PAS-stained 48-h kidney tissue sections. e Representative examples of PAS and NGAL staining of 48-h kidney sections. Arrows show tubular dilatation, cast and necrosis. NGAL expression

models of sepsis and cell therapies even with close attention to principles of good experimental design $[52,53]$ and that reflects similar challenges faced in the clinical application of novel therapies to sepsis [18, 20, 41, 54, 55].

Some limitations of the study should be acknowledged. In the first place, we have focussed on investigating the in vivo effects of CD362-selected hUC-MSC as this cell product is undergoing clinical trial for other inflammationdriven diseases including COVID-19-associated ARDS. showed peritubular appearance in the cortex of the kidney. Magnification 10X. Bar $=100 \mu \mathrm{m}$. Group sizes: SHAM+Saline+Saline $(n=9), \mathrm{CLP}+$ Saline+Saline $(n=8), \mathrm{CLP}+\mathrm{UC}-\mathrm{MSC}+$ Saline $(n=6)$ and CLP+UC-MSC+UC-MSC $(n=10)$. Statistical analysis: (A-B) One-way ANOVA with Bonferroni multiple comparison post-test and with 95\% confidence interval (C) Two-tailed Spearman correlative testing with regression line (D) Kruskal-Wallis test, Dunn's multiple comparison test, $p<0.05$. *Significantly different from SHAM group $\left({ }^{*} p<0.05, * * p<0.01, * * * p<0.001\right)$. \#Significantly different from CLP group $(p<0.05)$

This study design precluded gaining further insight into the comparative effects, in sepsis and SA-AKI, of the cell product tested with those of unselected hUC-MSC or with MSC derived from bone marrow or other tissues. Secondly, as the cell doses used were chosen on the basis of prior studies of human MSC anti-inflammatory effects in mice, it is not possible to determine whether multiple administrations of higher or lower cell numbers provides 
A

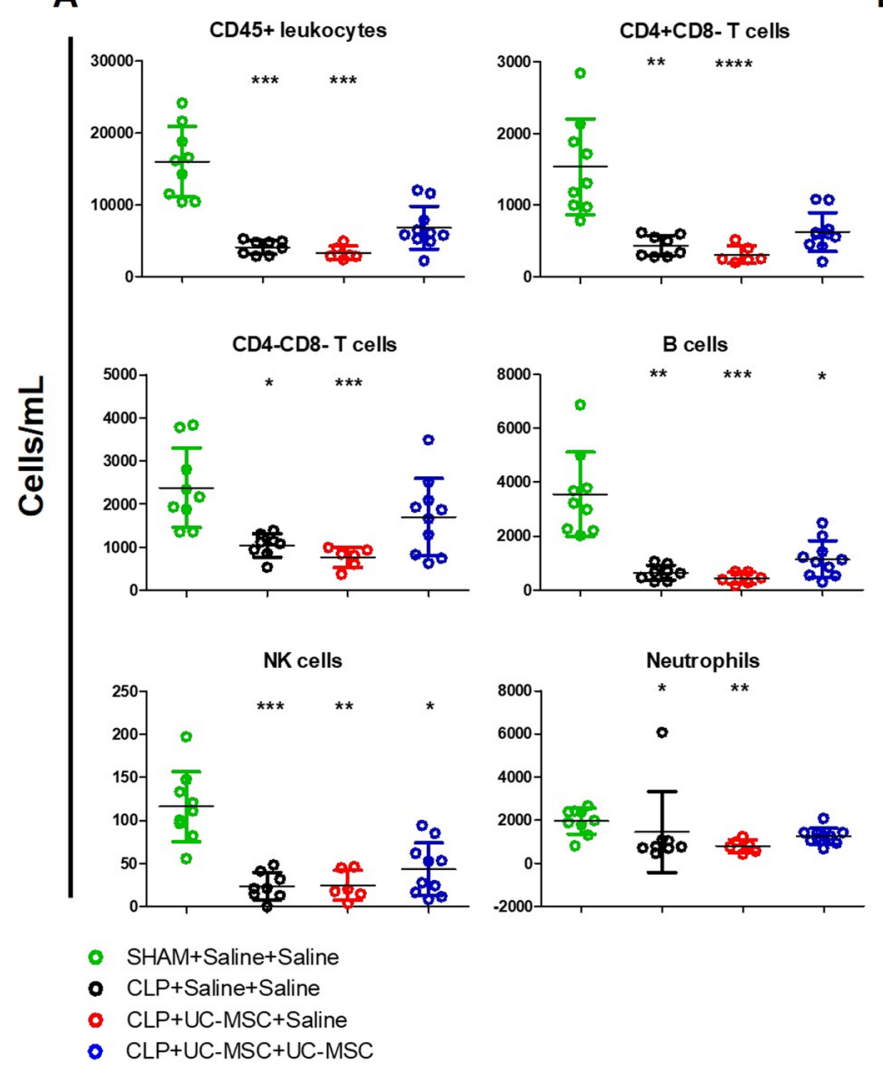

B

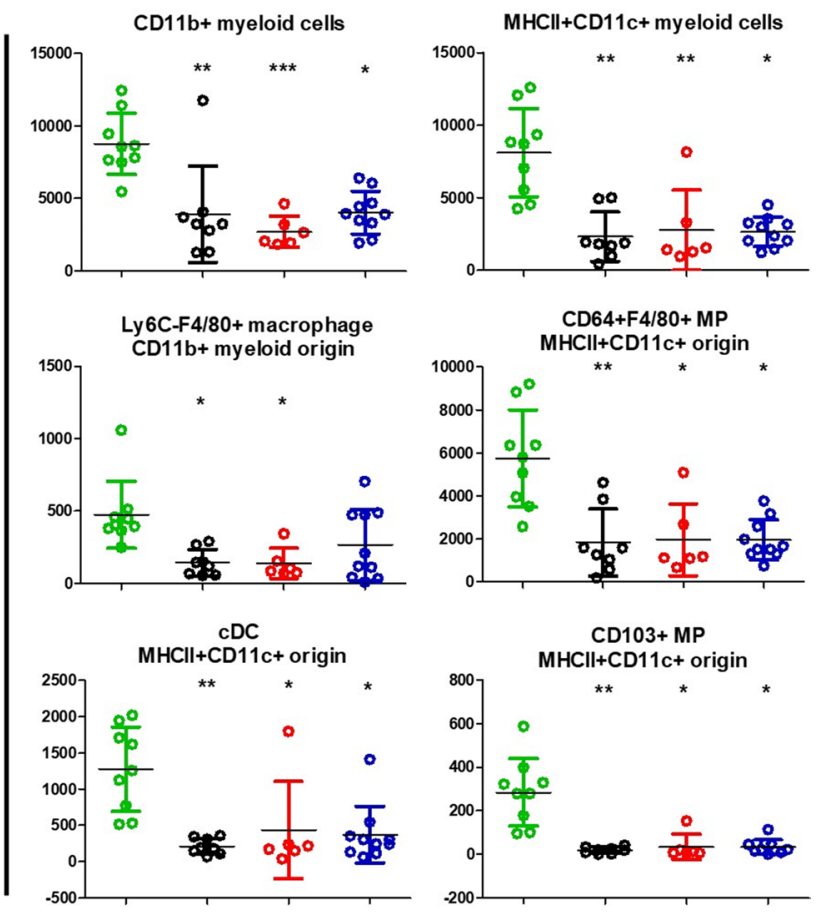

Fig. 5 Flow cytometric analysis of intra-renal immune cell subpopulations at 48 h following Sham or CLP procedures with saline, singledose hUC-MSC and double-dose hUC-MSC: Total cell numbers of intra-renal a lymphoid and $\mathbf{b}$ myeloid cell subpopulations quantified by multi-colour flow cytometry of CD45-enriched cell suspensions from collagenase/DNase-digested kidneys prepared at $48 \mathrm{~h}$ postprocedure from four groups of mice: SHAM+Saline+Saline $(n=9)$,
CLP + Saline+Saline $(n=8), \quad$ CLP + UC-MSC + Saline $(n=6)$ and CLP + UC-MSC + UC-MSC $(n=10)$. Statistical analysis: Non-Gaussian distribution: Kruskal-Wallis test with Dunn's multiple comparison test, $\mathrm{p}<0.05$. Gaussian distribution: One-way ANOVA with Bonferroni multiple comparison post-test and with $95 \%$ confidence interval. $*$ Significantly different from SHAM group $(* p<0.05, * * p<0.01$, $* * * p<0.001)$. Abbreviations: $\mathrm{MP}=$ mononuclear phagocytes greater benefit. Finally, as the group sizes for the CLP model experiments were powered to address hUC-MSC effects on a systemic inflammatory biomarker of sepsis/ SA-AKI severity, it is possible that larger group sizes would have more clearly defined the effect of repeated doses on survival. While these issues further emphasize the need for sequential pre-clinical experiments that adhere as closely as possible to the key parameters required for optimal clinical trial design, the results we present here support a continued focus on multi-dose regimens of antiinflammatory MSC in sepsis and SA-AKI.

Supplementary Information The online version contains supplementary material available at https://doi.org/10.1007/s12015-021-10323-7.

Acknowledgements The authors wish to acknowledge the assistance of Dr. Shirley Hanley who provided training and technical support for all flow cytometry analyses and of Dr. Yolanda Garcia for training and consultation in regard to animal model development and animal care and monitoring.

Authors' Contributions BF contributed to conception and design, performing experiments, data analysis and interpretation, provided financial support and drafted the manuscript. SA contributed to conception and design, performing experiments, data analysis and interpretation. LW, ON, CM and NN contributed to experimental procedure. JQG performed data analysis and interpretation. CC and MVA contributed to experimental procedures and data analysis. FL provided study material. SJE and MG contributed to conception and design, data analysis and interpretation and provided financial support. All authors read and approved the final manuscript.

Funding Open Access funding provided by the IReL Consortium. BF was supported by an Irish Research Council Enterprise Partnership Postdoctoral Fellowship (grant number EPSPD/2017/106), The Company of Biologists and Orbsen Therapeutics Ltd. MDG is supported by grants from the European Commission [Horizon 2020 Collaborative Health Project NEPHSTROM (grant number 634086) and FP7 Collaborative Health Project VISICORT (grant number 602470)], from Science Foundation Ireland [REMEDI Strategic Research Cluster 

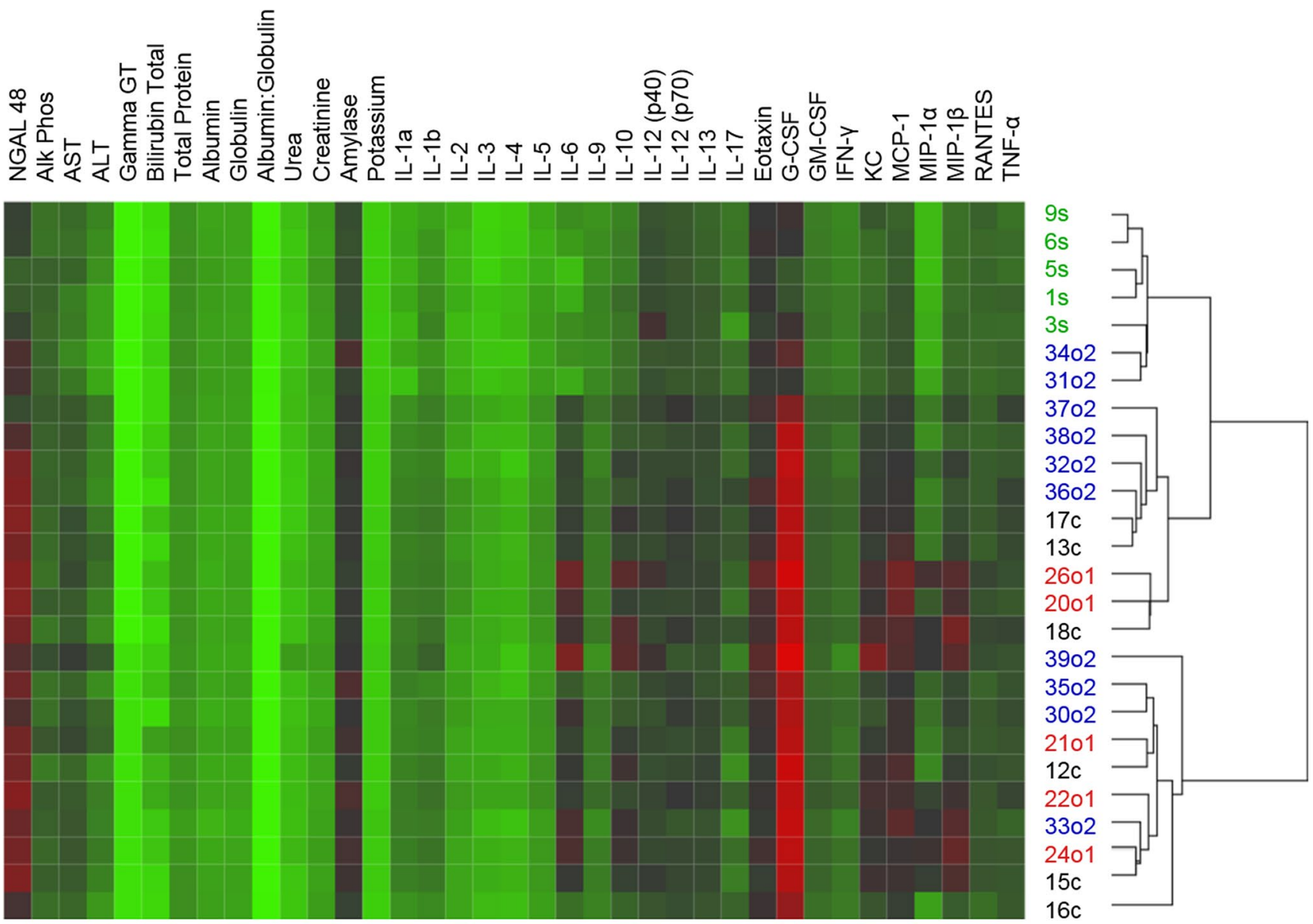

0

10 20

Fig. 6 Unsupervised hierarchical clustering of response patterns of control and hUC-MSC-treated animals: Average linkage Euclidean distance clustering of data from Sham (green, s, $n=5$ ), CLP (black, c, $n=6$ ) and UC-MSC-treated animals with single (red, o1, $n=5$ ) or double dose (blue, o2, $n=10$ ) of $1 \times 10^{6}$ UC-MSC. The lowest level is represented by green, while highest level is represented by red. All data except NGAL and Albumin:Globulin subjected to Log2 transformation prior to clustering (grant number 09/SRC-B1794) and CÚRAM Research Centre (grant number 13/RC/2073_P2) and the European Regional Development Fund. CMC was supported by an Irish Research Council Postgraduate Enterprise Partnership Grant (grant number EPSPG/2015/88). ON was supported by BrainMatTrain, part of the European Union Horizon 2020 Programme (H2020-MSCA-ITN-2015) under the Marie SkłodowskaCurie Initial Training Network and Grant Agreement No. 676408. FL is supported by Spanish Ministerio de Economía y Competitividad (MINECO; grants SAF-2016-80535-R and PID2019-106658RBI00) co-financed by European Development Regional Fund "A way to achieve Europe". CC and MVA are recipients of fellowships from MINECO (BES-2017-082107 and BES-2014-069237, respectively). Flow cytometry experiments were performed in the NUI Galway Flow Cytometry Core Facility which is supported by funds from NUI Galway, Science Foundation Ireland, the Irish Government's Programme for Research in Third Level Institutions, Cycle 5 and the European Regional Development Fund.
Data Availability The data that support the findings of this study are available from the corresponding author upon reasonable request.

\section{Declarations}

Ethics Approval All animal procedures were carried out under the license (no. 255/17) from the Animal Experimentation Ethical Committee, University of Barcelona and under authorisation (AE19125/ P082 and AE19125/P066) from the Health Products Regulatory Authority, Ireland, and approved by the NUI Galway Animal Care Research Ethics Committee. All procedures were performed in licensed animal facilities at NUI Galway and University of Barcelona.

Consent to Participate Not applicable. 
Consent for Publication "The pre-print version of this article is present on https://www.researchsquare.com/article/rs-547210/v1. This article is not published nor is under publication elsewhere."

Competing Interests $\mathrm{BF}, \mathrm{NN}$, JQG CC, MVA and MDG have no potential conflicts of interest to declare. SA, LW, ON, CMC and SJE are paid employees of Orbsen Therapeutics Ltd., a cell therapy company that is developing CD362-selected human umbilical cord-derived MSC for clinical applications. FL is founder and ad-honorem scientific advisor of Sepsia Therapeutics.

Open Access This article is licensed under a Creative Commons Attribution 4.0 International License, which permits use, sharing, adaptation, distribution and reproduction in any medium or format, as long as you give appropriate credit to the original author(s) and the source, provide a link to the Creative Commons licence, and indicate if changes were made. The images or other third party material in this article are included in the article's Creative Commons licence, unless indicated otherwise in a credit line to the material. If material is not included in the article's Creative Commons licence and your intended use is not permitted by statutory regulation or exceeds the permitted use, you will need to obtain permission directly from the copyright holder. To view a copy of this licence, visit http://creativecommons.org/licenses/by/4.0/.

\section{References}

1. Singer, M., Deutschman, C. S., Seymour, C. W., Shankar-Hari, M., Annane, D., Bauer, M., Bellomo, R., Bernard, G. R., Chiche, J. D., Coopersmith, C. M., Hotchkiss, R. S., Levy, M. M., Marshall, J. C., Martin, G. S., Opal, S. M., Rubenfeld, G. D., van der Poll, T., Vincent, J. L., \& Angus, D. C. (2016). The third international consensus definitions for sepsis and septic shock (sepsis-3). The Journal of the American Medical Associatio, 315(8), 801-810. https://doi.org/10.1001/jama.2016.0287

2. Hoste, E. A., Bagshaw, S. M., Bellomo, R., Cely, C. M., Colman, R., Cruz, D. N., Edipidis, K., Forni, L. G., Gomersall, C. D., Govil, D., Honore, P. M., Joannes-Boyau, O., Joannidis, M., Korhonen, A. M., Lavrentieva, A., Mehta, R. L., Palevsky, P., Roessler, E., Ronco, C., et al. (2015). Epidemiology of acute kidney injury in critically ill patients: The multinational AKI-EPI study. Intensive Care Medicine, 41(8), 1411-1423. https://doi.org/ 10.1007/s00134-015-3934-7

3. Rudd, K. E., Johnson, S. C., Agesa, K. M., Shackelford, K. A., Tsoi, D., Kievlan, D. R., Colombara, D. V., Ikuta, K. S., Kissoon, N., Finfer, S., Fleischmann-Struzek, C., Machado, F. R., Reinhart, K. K., Rowan, K., Seymour, C. W., Watson, R. S., West, T. E., Marinho, F., Hay, S. I., et al. (2020). Global, regional, and national sepsis incidence and mortality, 1990-2017: Analysis for the global burden of disease study. Lancet, 395(10219), 200-211. https://doi. org/10.1016/S0140-6736(19)32989-7

4. Arutyunyan, I., Elchaninov, A., Makarov, A., \& Fatkhudinov, T. (2016). Umbilical cord as prospective source for mesenchymal stem cell-based therapy. Stem Cells International, 2016, 6901286. https://doi.org/10.1155/2016/6901286

5. Fazekas, B., \& Griffin, M. D. (2020). Mesenchymal stromal cellbased therapies for acute kidney injury: Progress in the last decade. Kidney International, 97(6), 1130-1140. https://doi.org/10. 1016/j.kint.2019.12.019

6. Hickson, L. J., Herrmann, S. M., McNicholas, B. A., \& Griffin, M. D. (2021). Progress toward the clinical application of mesenchymal stromal cells and other disease-modulating regenerative therapies: Examples from the field of nephrology. Kidney360, 2(3), 542-557. https://doi.org/10.34067/kid.0005692020

7. Weiss, A. R. R., Lee, O., Eggenhofer, E., Geissler, E., Korevaar, S. S., Soeder, Y., Schlitt, H. J., Geissler, E. K., Hoogduijn, M. J., \& Dahlke, M. H. (2020). Differential effects of heat-inactivated, secretome-deficient msc and metabolically active msc in sepsis and allogenic heart transplantation. Stem Cells, 38(6), 797-807. https://doi.org/10.1002/stem.3165

8. Jerkic, M., Gagnon, S., Rabani, R., Ward-Able, T., Masterson, C., Otulakowski, G., Curley, G. F., Marshall, J., Kavanagh, B. P., \& Laffey, J. G. (2020). Human umbilical cord mesenchymal stromal cells attenuate systemic sepsis in part by enhancing peritoneal macrophage bacterial killing via heme oxygenase- 1 induction in rats. Anesthesiology, 132(1), 140-154. https://doi.org/10.1097/ ALN.0000000000003018

9. Wu, K. H., Wu, H. P., Chao, W. R., Lo, W. Y., Tseng, P. C., Lee, C. J., Peng, C. T., Lee, M. S., \& Chao, Y. H. (2016). Time-series expression of toll-like receptor 4 signaling in septic mice treated with mesenchymal stem cells. Shock, 45(6), 634-640. https://doi. org/10.1097/SHK.0000000000000546

10. Chang, C. L., Leu, S., Sung, H. C., Zhen, Y. Y., Cho, C. L., Chen, A., Tsai, T. H., Chung, S. Y., Chai, H. T., Sun, C. K., Yen, C. H., \& Yip, H. K. (2012). Impact of apoptotic adipose-derived mesenchymal stem cells on attenuating organ damage and reducing mortality in rat sepsis syndrome induced by cecal puncture and ligation. Journal of Translational Medicine, 10, 244. https://doi. org/10.1186/1479-5876-10-244

11. Hall, S. R., Tsoyi, K., Ith, B., Padera Jr., R. F., Lederer, J. A., Wang, Z., Liu, X., \& Perrella, M. A. (2013). Mesenchymal stromal cells improve survival during sepsis in the absence of heme oxygenase-1: The importance of neutrophils. Stem Cells, 31(2), 397-407. https://doi.org/10.1002/stem.1270

12. Zhang, G., Zou, X., Huang, Y., Wang, F., Miao, S., Liu, G., Chen, M., \& Zhu, Y. (2016). Mesenchymal stromal cell-derived extracellular vesicles protect against acute kidney injury through anti-oxidation by enhancing nrf2/are activation in rats. Kidney and Blood Pressure Research, 41(2), 119-128. https://doi.org/10. $1159 / 000443413$

13. Luo, C. J., Zhang, F. J., Zhang, L., Geng, Y. Q., Li, Q. G., Hong, Q., Fu, B., Zhu, F., Cui, S. Y., Feng, Z., Sun, X. F., \& Chen, X. M. (2014). Mesenchymal stem cells ameliorate sepsis-associated acute kidney injury in mice. Shock, 41(2), 123-129. https://doi. org/10.1097/SHK.0000000000000080

14. Condor, J. M., Rodrigues, C. E., Sousa Moreira, R., Canale, D., Volpini, R. A., Shimizu, M. H., Camara, N. O., Noronha Ide, L., \& Andrade, L. (2016). Treatment with human wharton's jellyderived mesenchymal stem cells attenuates sepsis-induced kidney injury, liver injury, and endothelial dysfunction. Stem Cells Translational Medicine, 5(8), 1048-1057. https://doi.org/10.5966/sctm. 2015-0138

15. Zullo, J. A., Nadel, E. P., Rabadi, M. M., Baskind, M. J., Rajdev, M. A., Demaree, C. M., Vasko, R., Chugh, S. S., Lamba, R., Goligorsky, M. S., \& Ratliff, B. B. (2015). The secretome of hydrogelcoembedded endothelial progenitor cells and mesenchymal stem cells instructs macrophage polarization in endotoxemia. Stem Cells Translational Medicine, 4(7), 852-861. https://doi.org/10. 5966/sctm.2014-0111

16. Perlee, D., van Vught, L. A., Scicluna, B. P., Maag, A., Lutter, R., Kemper, E. M., van 't Veer, C., Punchard, M. A., Gonzalez, J., Richard, M. P., Dalemans, W., Lombardo, E., de Vos, A. F., \& van der Poll, T. (2018). Intravenous infusion of human adipose mesenchymal stem cells modifies the host response to lipopolysaccharide in humans: A randomized, single-blind, parallel group, placebo controlled trial. Stem Cells, 36(11), 1778-1788. https:// doi.org/10.1002/stem.2891 
17. Gennadiy Galstyan, P. M., Parovichnikova, E., Kuzmina, L., Troitskaya, V., Gemdzhian, E., \& Savchenko, V. (2018). The results of the single center pilot randomized Russian clinical trial of mesenchymal stromal cells in severe neutropenic patients with septic shock (RUMCESS). International Journal Blood Research and Disorders, 5(1), 33. https://doi.org/10.23937/2469-5696/1410033

18. McIntyre, L. A., Stewart, D. J., Mei, S. H. J., Courtman, D., Watpool, I., Granton, J., Marshall, J., Dos Santos, C., Walley, K. R., Winston, B. W., Schlosser, K., Fergusson, D. A., \& Canadian Critical Care Trials Group, \& Canadian Critical Care Translational Biology Group. (2018). Cellular immunotherapy for septic shock. A phase I clinical trial. American Journal of Respiratory and Critical Care Medicine, 197(3), 337-347. https://doi.org/10. 1164/rccm.201705-1006OC

19. Matthay, M. A., Calfee, C. S., Zhuo, H., Thompson, B. T., Wilson, J. G., Levitt, J. E., Rogers, A. J., Gotts, J. E., Wiener-Kronish, J. P., Bajwa, E. K., Donahoe, M. P., McVerry, B. J., Ortiz, L. A., Exline, M., Christman, J. W., Abbott, J., Delucchi, K. L., Caballero, L., McMillan, M., et al. (2019). Treatment with allogeneic mesenchymal stromal cells for moderate to severe acute respiratory distress syndrome (START study): A randomised phase 2a safety trial. The Lancet Respiratory Medicine, 7(2), 154-162. https://doi.org/10. 1016/S2213-2600(18)30418-1

20. Swaminathan, M., Stafford-Smith, M., Chertow, G. M., Warnock, D. G., Paragamian, V., Brenner, R. M., Lellouche, F., FoxRobichaud, A., Atta, M. G., Melby, S., Mehta, R. L., Wald, R., Verma, S., Mazer, C. D., \& investigators, A.-A. (2018). Allogeneic mesenchymal stem cells for treatment of AKI after cardiac surgery. Journal of the American Society of Nephrology, 29(1), 260-267. https://doi.org/10.1681/ASN.2016101150

21. Gotts, J. E., \& Matthay, M. A. (2018). Cell-based therapy in sepsis. A step closer. American Journal of Respiratory and Critical Care Medicine, 197(3), 280-281. https://doi.org/10.1164/rccm. 201710-2068ED

22. Griffin, M. D., \& Swaminathan, S. (2020). Editorial: Innovative biologics and drugs to target renal inflammation. Frontiers in Pharmacology, 11, 38. https://doi.org/10.3389/fphar.2020.00038

23. Horie, S., Masterson, C., Brady, J., Loftus, P., Horan, E., O'Flynn, L., Elliman, S., Barry, F., O’Brien, T., Laffey, J. G., \& O’Toole, D. (2020). Umbilical cord-derived CD362(+) mesenchymal stromal cells for e. coli pneumonia: Impact of dose regimen, passage, cryopreservation, and antibiotic therapy. Stem Cell Research \& Therapy, 11(1), 116. https://doi.org/10.1186/s13287-020-01624-8

24. Gonzalez, H., Keane, C., Masterson, C. H., Horie, S., Elliman, S. J., Higgins, B. D., Scully, M., Laffey, J. G., \& O’Toole, D. (2020). Umbilical cord-derived CD362(+) mesenchymal stromal cells attenuate polymicrobial sepsis induced by caecal ligation and puncture. International Journal of Molecular Sciences, 21(21). https://doi.org/10.3390/ijms21218270

25. Lewis, A. J., Seymour, C. W., \& Rosengart, M. R. (2016). Current murine models of sepsis. Surgical Infections, 17(4), 385-393. https://doi.org/10.1089/sur.2016.021

26. Remick, D. G., \& Ward, P. A. (2005). Evaluation of endotoxin models for the study of sepsis. Shock, 24(Suppl 1), 7-11. https:// doi.org/10.1097/01.shk.0000191384.34066.85

27. de Witte, S. F. H., Luk, F., Sierra Parraga, J. M., Gargesha, M., Merino, A., Korevaar, S. S., Shankar, A. S., O'Flynn, L., Elliman, S. J., Roy, D., Betjes, M. G. H., Newsome, P. N., Baan, C. C., \& Hoogduijn, M. J. (2018). Immunomodulation by therapeutic mesenchymal stromal cells (MSC) is triggered through phagocytosis of msc by monocytic cells. Stem Cells, 36(4), 602-615. https:// doi.org/10.1002/stem.2779

28. Zhao, X., Liu, D., Gong, W., Zhao, G., Liu, L., Yang, L., \& Hou, Y. (2014). The toll-like receptor 3 ligand, poly(i:C), improves immunosuppressive function and therapeutic effect of mesenchymal stem cells on sepsis via inhibiting mir-143. Stem Cells, 32(2), 521-533. https://doi.org/10.1002/stem.1543

29. Nemeth, K., Leelahavanichkul, A., Yuen, P. S., Mayer, B., Parmelee, A., Doi, K., Robey, P. G., Leelahavanichkul, K., Koller, B. H., Brown, J. M., Hu, X., Jelinek, I., Star, R. A., \& Mezey, E. (2009). Bone marrow stromal cells attenuate sepsis via prostaglandin E(2)-dependent reprogramming of host macrophages to increase their interleukin-10 production. Nature Medicine, 15(1), 42-49. https://doi.org/10.1038/nm.1905

30. Fletcher, A. L., Elman, J. S., Astarita, J., Murray, R., Saeidi, N., D’Rozario, J., Knoblich, K., Brown, F. D., Schildberg, F. A., Nieves, J. M., Heng, T. S., Boyd, R. L., Turley, S. J., \& Parekkadan, B. (2014). Lymph node fibroblastic reticular cell transplants show robust therapeutic efficacy in high-mortality murine sepsis. Science Translational Medicine, 6(249), 249ra109. https:// doi.org/10.1126/scitranslmed.3009377

31. Gonzalez-Rey, E., Anderson, P., Gonzalez, M. A., Rico, L., Buscher, D., \& Delgado, M. (2009). Human adult stem cells derived from adipose tissue protect against experimental colitis and sepsis. Gut, 58(7), 929-939. https://doi.org/10.1136/gut.2008. 168534

32. Kaucsar, T., Godo, M., Revesz, C., Kovacs, M., Mocsai, A., Kiss, N., Albert, M., Krenacs, T., Szenasi, G., \& Hamar, P. (2016). Urine/plasma neutrophil gelatinase associated lipocalin ratio is a sensitive and specific marker of subclinical acute kidney injury in mice. PLoS One, 11(1), e0148043. https://doi.org/10.1371/journ al.pone. 0148043

33. Park, H. K., Kim, S., Lee, S.-S., Kim, H.-J., Ahn, H.-S., Song, J. Y., Um, T.-H., Cho, C.-R., Jung, H., Koo, H.-K., Sim, Y. S., Song, J.-U., \& Park, J.-H. (2015). Does plasma NGAL help for prediction of acute kidney injury in sepsis patients?: A systematic review and meta-analysis. European Respiratory Journal, 46(suppl 59), PA2148. https://doi.org/10.1183/13993003.congr ess-2015.PA2148

34. Koeze, J., van der Horst, I. C. C., Keus, F., Wiersema, R., Dieperink, W., Kootstra-Ros, J. E., Zijlstra, J. G., \& van Meurs, M. (2020). Plasma neutrophil gelatinase-associated lipocalin at intensive care unit admission as a predictor of acute kidney injury progression. Clinical Kidney Journal, 13(6), 994-1002. https://doi. org/10.1093/ckj/sfaa002

35. Lee, K. Y. (2019). M1 and M2 polarization of macrophages: A mini-review. Medical Biological Science and Engineering, 2(1), 1-5. https://doi.org/10.30579/mbse.2019.2.1.1

36. Eggenhofer, E., Luk, F., Dahlke, M. H., \& Hoogduijn, M. J. (2014). The life and fate of mesenchymal stem cells. Frontiers in Immunology, 5, 148. https://doi.org/10.3389/fimmu.2014.00148

37. Luk, F., de Witte, S. F., Korevaar, S. S., Roemeling-van Rhijn, M., Franquesa, M., Strini, T., van den Engel, S., Gargesha, M., Roy, D., Dor, F. J., Horwitz, E. M., de Bruin, R. W., Betjes, M. G., Baan, C. C., \& Hoogduijn, M. J. (2016). Inactivated mesenchymal stem cells maintain immunomodulatory capacity. Stem Cells and Development, 25(18), 1342-1354. https://doi.org/10.1089/scd. 2016.0068

38. Li, J. L., Li, G., Jing, X. Z., Li, Y. F., Ye, Q. Y., Jia, H. H., Liu, S. H., Li, X. J., Li, H., Huang, R., Zhang, Y., \& Wang, H. (2018). Assessment of clinical sepsis-ssociated biomarkers in a septic mouse model. Journal of International Medical Research, 46(6), 2410-2422. https://doi.org/10.1177/0300060518764717

39. Cai, L., Rubin, J., Han, W., Venge, P., \& Xu, S. (2010). The origin of multiple molecular forms in urine of HNL/NGAL. Clinical Journal of the American Society of Nephrology, 5(12), 22292235. https://doi.org/10.2215/CJN.00980110

40. Capcha, J. M. C., Rodrigues, C. E., Moreira, R. S., Silveira, M. D., Dourado, P., Dos Santos, F., Irigoyen, M. C., Jensen, L., Garnica, M. R., Noronha, I. L., Andrade, L., \& Gomes, S. A. (2020). 
Wharton's jelly-derived mesenchymal stem cells attenuate sepsisinduced organ injury partially via cholinergic anti-inflammatory pathway activation. American Journal of Physiology. Regulatory, Integrative and Comparative Physiology, 318(1), R135-R147. https://doi.org/10.1152/ajpregu.00098.2018

41. Schlosser, K., Wang, J. P., Dos Santos, C., Walley, K. R., Marshall, J., Fergusson, D. A., Winston, B. W., Granton, J., Watpool, I., Stewart, D. J., McIntyre, L. A., Mei, S. H. J., \& Canadian Critical Care Trials, G., \& the Canadian Critical Care Translational Biology, G. (2019). Effects of mesenchymal stem cell treatment on systemic cytokine levels in a phase 1 dose escalation safety trial of septic shock patients. Critical Care Medicine, 47(7), 918925. https://doi.org/10.1097/CCM.0000000000003657

42. van Rhijn-Brouwer, F. C. C., Gremmels, H., Fledderus, J. O., \& Verhaar, M. C. (2018). Mesenchymal stromal cell characteristics and regenerative potential in cardiovascular disease: Implications for cellular therapy. Cell Transplantation, 27(5), 765-785. https:// doi.org/10.1177/0963689717738257

43. Sun, X. Y., Ding, X. F., Liang, H. Y., Zhang, X. J., Liu, S. H., Bing, H., Duan, X. G., \& Sun, T. W. (2020). Efficacy of mesenchymal stem cell therapy for sepsis: A meta-analysis of preclinical studies. Stem Cell Research \& Therapy, 11(1), 214. https://doi.org/ 10.1186/s13287-020-01730-7

44. Laroye, C., Gibot, S., Reppel, L., \& Bensoussan, D. (2017). Concise review: Mesenchymal stromal/stem cells: A new treatment for sepsis and septic shock? Stem Cells, 35(12), 2331-2339. https:// doi.org/10.1002/stem.2695

45. Brady, J., Horie, S., \& Laffey, J. G. (2020). Role of the adaptive immune response in sepsis. Intensive Care Medicine Experimental, 8(Suppl 1), 20. https://doi.org/10.1186/s40635-020-00309-z

46. Martina, M. N., Noel, S., Saxena, A., Bandapalle, S., Majithia, R., Jie, C., Arend, L. J., Allaf, M. E., Rabb, H., \& Hamad, A. R. (2016). Double-negative alpha-beta $T$ cells are early responders to Aki and are found in human kidney. Journal of the American Society of Nephrology, 27(4), 1113-1123. https://doi.org/10.1681/ ASN.2014121214

47. Hotchkiss, R. S., Tinsley, K. W., Swanson, P. E., Schmieg Jr., R. E., Hui, J. J., Chang, K. C., Osborne, D. F., Freeman, B. D., Cobb, J. P., Buchman, T. G., \& Karl, I. E. (2001). Sepsis-induced apoptosis causes progressive profound depletion of B and CD4+ $\mathrm{T}$ lymphocytes in humans. The Journal of Immunology, 166(11), 6952-6963. https://doi.org/10.4049/jimmunol.166.11.6952

48. Condotta, S. A., Cabrera-Perez, J., Badovinac, V. P., \& Griffith, T. S. (2013). T-cell-mediated immunity and the role of TRAIL in sepsis-induced immunosuppression. Critical Reviews in Immunology, 33(1), 23-40. https://doi.org/10.1615/critrevimmunol.20130 06721
49. Khosrojerdi, A., Soudi, S., Hosseini, A. Z., Eshghi, F., Shafiee, A., \& Hashemi, S. M. (2021). Immunomodulatory and therapeutic effects of mesenchymal stem cells on organ dysfunction in sepsis. Shock, 55(4), 423-440. https://doi.org/10.1097/SHK.0000000000 001644

50. Nie, P., Bai, X., Lou, Y., Zhu, Y., Jiang, S., Zhang, L., Tian, N., Luo, P., \& Li, B. (2021). Human umbilical cord mesenchymal stem cells reduce oxidative damage and apoptosis in diabetic nephropathy by activating Nrf2. Stem Cell Research \& Therapy, 12(1), 450. https://doi.org/10.1186/s13287-021-02447-x

51. dos Santos, C. C., Murthy, S., Hu, P., Shan, Y., Haitsma, J. J., Mei, S. H., Stewart, D. J., \& Liles, W. C. (2012). Network analysis of transcriptional responses induced by mesenchymal stem cell treatment of experimental sepsis. American Journal of Pathology, 181(5), 1681-1692. https://doi.org/10.1016/j.ajpath.2012.08.009

52. von Kortzfleisch, V. T., Karp, N. A., Palme, R., Kaiser, S., Sachser, N., \& Richter, S. H. (2020). Improving reproducibility in animal research by splitting the study population into several 'miniexperiments'. Scientific Reports, 10(1), 16579. https://doi.org/10. 1038/s41598-020-73503-4

53. Tanavde, V., Vaz, C., Rao, M. S., Vemuri, M. C., \& Pochampally, R. R. (2015). Research using mesenchymal stem/stromal cells: Quality metric towards developing a reference material. Cytotherapy, 17(9), 1169-1177. https://doi.org/10.1016/j.jcyt.2015. 07.008

54. Pickkers, P., Heemskerk, S., Schouten, J., Laterre, P. F., Vincent, J. L., Beishuizen, A., Jorens, P. G., Spapen, H., Bulitta, M., Peters, W. H., \& van der Hoeven, J. G. (2012). Alkaline phosphatase for treatment of sepsis-induced acute kidney injury: A prospective randomized double-blind placebo-controlled trial. Critical Care, 16(1), R14. https://doi.org/10.1186/cc11159

55. Pickkers, P., Mehta, R. L., Murray, P. T., Joannidis, M., Molitoris, B. A., Kellum, J. A., Bachler, M., Hoste, E. A. J., Hoiting, O., Krell, K., Ostermann, M., Rozendaal, W., Valkonen, M., Brealey, D., Beishuizen, A., Meziani, F., Murugan, R., de Geus, H., Payen, D., et al. (2018). Effect of human recombinant alkaline phosphatase on 7-day creatinine clearance in patients with sepsisassociated acute kidney injury: A randomized clinical trial. The Journal of the American Medical Association, 320(19), 19982009. https://doi.org/10.1001/jama.2018.14283

Publisher's Note Springer Nature remains neutral with regard to jurisdictional claims in published maps and institutional affiliations. 